Mélanges de l'École française de Rome Antiquité

131-2 | 2019

Fac-simile 1: le collezioni di documentazione grafica sulla pittura etrusca - Varia

\title{
Le ultime acquisizioni dal teatro di Terracina e l'eccezionale iscrizione del triumviro M. Emilio Lepido
}

Nicoletta Cassieri, Gian Luca Gregori e Jean-Baptiste Refalo-Bistagne

\section{OpenEdition}

\section{Edizione digitale}

URL: http://journals.openedition.org/mefra/9174

DOI: $10.4000 /$ mefra.9174

ISSN: $1724-2134$

\section{Editore}

École française de Rome

Edizione cartacea

ISBN: 978-2-7283-1430-0

ISSN: 0223-5102

\section{Notizia bibliografica digitale}

Nicoletta Cassieri, Gian Luca Gregori e Jean-Baptiste Refalo-Bistagne, « Le ultime acquisizioni dal teatro di Terracina e l'eccezionale iscrizione del triumviro M. Emilio Lepido », Mélanges de l'École française de Rome - Antiquité [Online], 131-2 | 2019, Messo online il 22 avril 2020, consultato il 30 avril 2020. URL : http://journals.openedition.org/mefra/9174 ; DOI : https://doi.org/10.4000/mefra.9174

Questo documento è stato generato automaticamente il 30 aprile 2020.

(c) École française de Rome 


\title{
Le ultime acquisizioni dal teatro di Terracina e l'eccezionale iscrizione del triumviro M. Emilio Lepido
}

\author{
Nicoletta Cassieri, Gian Luca Gregori e Jean-Baptiste Refalo-Bistagne
}

\section{NOTE DELL'AUTORE}

Gli autori ringraziano Bertrand Augier (École française de Rome), Clément Chillet (Université Grenoble-Alpes) e Francesca Rohr (Università Ca' Foscari Venezia), per gli utili consigli dati nella stesura finale del loro lavoro.

\section{Vent'anni di ricerche nell'area del teatro di Terracina}

\section{$1 \quad$ Nicoletta Cassieri}

Tra gli antichi centri del Lazio con continuità di vita fino ai nostri giorni, Terracina (Anxur-Tarracina) vanta un sottosuolo archeologico tuttora di straordinaria consistenza come documentano le scoperte degli ultimi decenni (strade, ville, impianti termali, horrea, domus) sia nella città «alta» sia nella città «bassa»1. Esse si affiancano alle testimonianze censite in maniera sistematica dal Lugli ${ }^{2}$, contribuendo ad arricchire il quadro topografico antico ed evidenziando l'alto grado di benessere raggiunto dalla colonia repubblicana, dotata di un porto e situata in posizione strategica su un avancorpo dei Monti Ausoni che qui giungevano al mare, al centro di percorsi marittimi e di assi viari, tra cui la via Appia, essenziale arteria di comunicazione tra Roma, l'Italia meridionale e l'Oriente. L'afflusso di ricchezze derivanti dai traffici mediterranei e l'affermarsi dei ceti imprenditoriali determinarono già nella seconda metà del II-inizi 
I sec. a.C. l'avvio di una prima significativa sistemazione urbanistica del Foro (oggi Piazza Municipio) $)^{3}$ e lo scenografico rinnovamento dell'antichissimo santuario di Monte S. Angelo, uno dei grandi complessi sacri del Lazio repubblicano.

Indipendentemente dall'ipotizzata deduzione di una colonia triumvirale ${ }^{4}$ tra la fine della Repubblica e la prima età imperiale, Tarracina, come altri centri costieri del sinus Amyclanus, fu interessata da un'intensa attività edilizia ${ }^{5}$, riguardante innanzitutto la ricostruzione monumentale del vecchio Foro che, ampliato attraverso un sistema di gallerie sostruttive, vide gli edifici circostanti la piazza ristrutturati o eretti ex novo (il Tempio Maggiore, il complesso del teatro-portico, la basilica, i portici e gli imponenti archi di accesso) ${ }^{6}$. La pavimentazione in lastre di calcare, conservata per notevole estensione, reca tuttora i solchi dell'iscrizione dedicatoria con i fori per l'alloggiamento di grandi lettere in bronzo menzionanti un Aulus Aemilius A.f., non meglio noto, probabile finanziatore dell'opera ${ }^{7}$.

3 A questi progetti di riqualificazione degli spazi pubblici, che richiesero ingenti risorse finanziarie, non rimasero estranei notabili locali ed esponenti di famiglie urbane di alto rango, in parte originarie della zona, dove avevano importanti interessi economici, proprietà fondiarie e ville imponenti (in particolare gli Aemilii Lepidi e i Sulpicii Galbae) ${ }^{8}$.

4 L'impegnativo piano edilizio e monumentale destinato a cambiare radicalmente l'aspetto della città, che ricevette speciale impulso in età augustea, con ogni evidenza fu attuato per gradi e portato a conclusione non prima della metà del I sec. d.c. (fig. 1). L'elevata qualità degli interventi architettonici e dei programmi decorativi, oltre alla profusione di materiali di pregio precocemente utilizzati, lasciano presumere l'attività di maestranze specializzate, forse di formazione urbana o campana, esperte del nuovo linguaggio formale, che collaborarono al processo di adeguamento degli edifici ai modelli di Roma, promosso da una classe dirigente desiderosa di manifestare lealismo nei confronti del potere centrale e adesione ai valori del principato 9 . 
Fig. 1 - Piazza Municipio. Il Foro Emiliano, visto dall'alto.

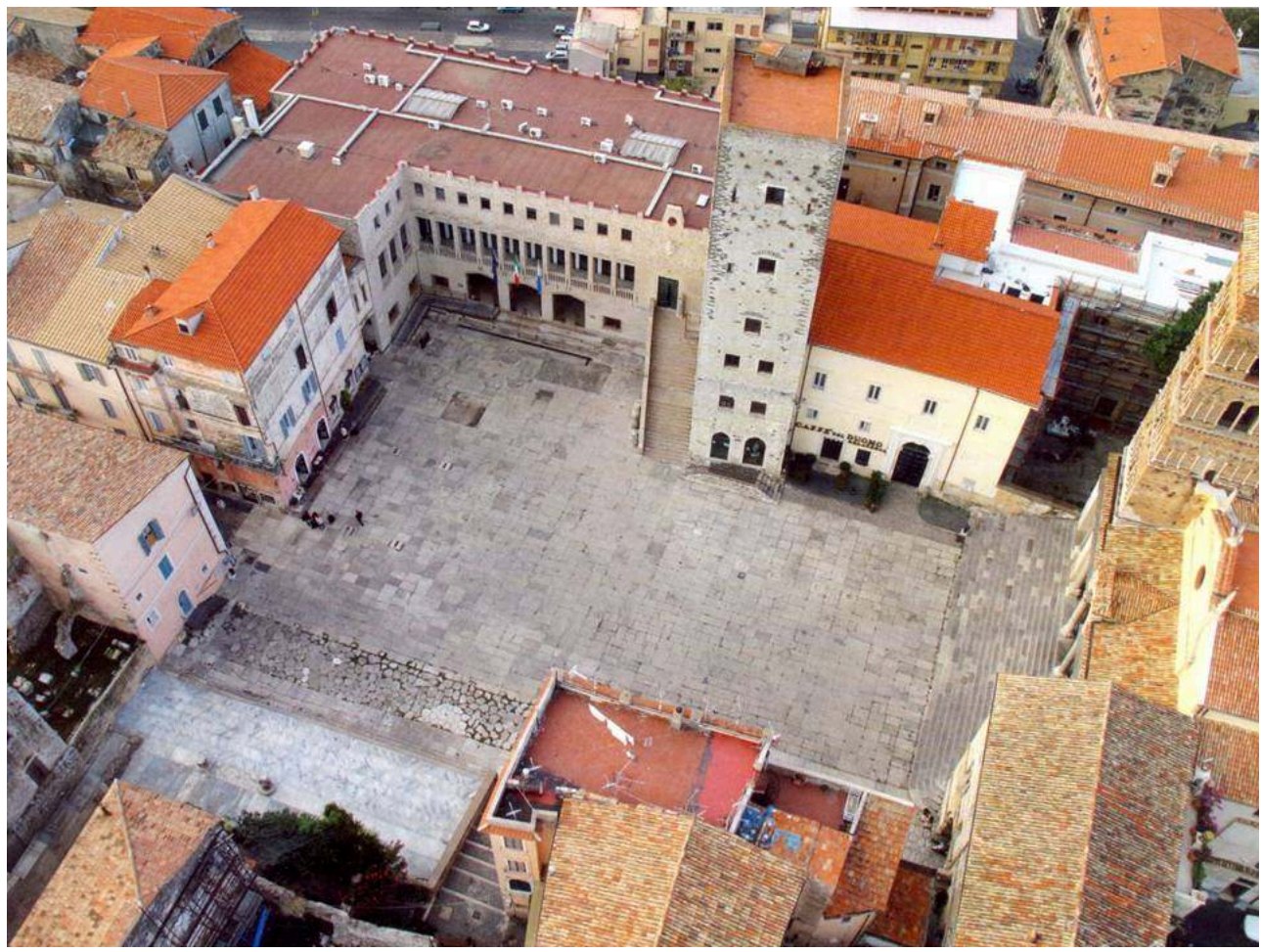

Foto N. Cassieri.

5 L'edificio teatrale viene a occupare un'ampia superficie $(70 \times 60 \mathrm{~m})$, tangente il tratto urbano della via Appia, sul versante settentrionale del complesso forense che rappresenta un episodio di conservazione archeologica e urbanistica di singolare rarità (fig. 2). Piuttosto che dalla volontà di incidere su un settore cittadino, all'epoca evidentemente non del tutto edificato, la sua ubicazione fu determinata dalle caratteristiche morfologiche del luogo, capace di accogliere l'invaso della cavea che si appoggiò, almeno in parte, al pendio naturale, consentendo in tal modo un notevole risparmio di lavoro e di forniture edilizie. Tale area costituisce quindi, a tutti gli effetti, parte integrante del cd. Foro Emiliano, fulcro della vita politica, religiosa ed economica della città che ne ha perpetuato valore e funzioni fino ai nostri giorni. 
Fig. 2 - Foto con evidenziazione dei diversi settori del teatro e dell'area forense.

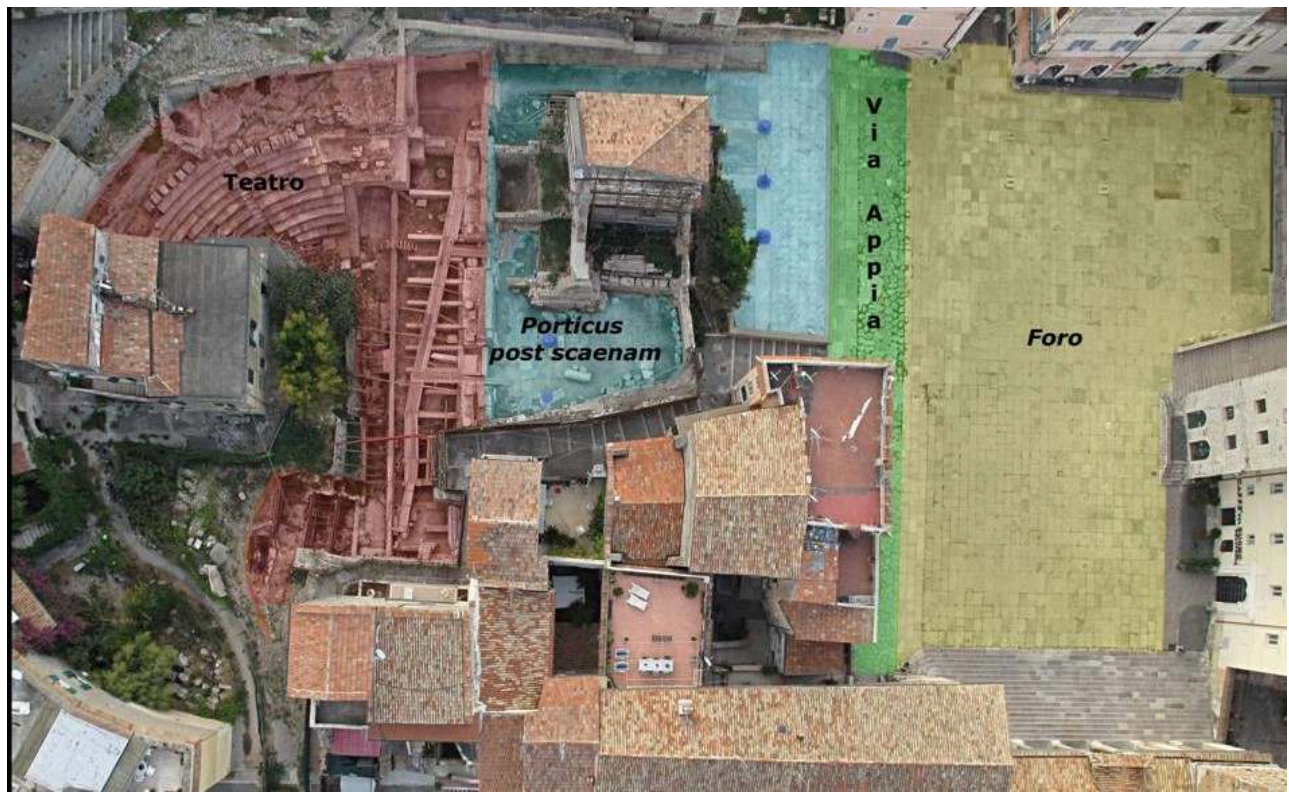

Foto N. Cassieri.

6 La conoscenza del monumento risale ad anni recenti: dopo i devastanti bombardamenti del 1943 che colpirono anche il popolare quartiere incentrato sulla piccola Piazza Urbano II, l'abbattimento delle strutture pericolanti e lo sgombero delle macerie fecero affiorare alcuni resti murari, che vennero attribuiti ipoteticamente a un edificio per spettacoli ${ }^{10}$. Il progressivo abbandono dell'isolato da parte dei residenti ha determinato una situazione di stasi e di crescente degrado del tutto inusuale in un abitato di secolare vitalità, consentendo, d'altro canto, di avviare agli inizi del Duemila un piano organico di indagini archeologiche e di restauri, che hanno comportato la rimozione «stratigrafica» di fatiscenti fabbricati moderni e di diffuse superfetazioni.

Del notevole complesso formato dal teatro e dalla retrostante porticus post scaenam, sono stati riportati in luce ampi settori che consentono di tracciare, almeno nelle linee generali, le sue vicende costruttive; circa un terzo dell'edificio, tuttavia, resta al momento sconosciuto, data l'insistenza di un denso tessuto insediativo sulla parte occidentale $^{11}$. Dagli elementi accertati è possibile fissare intorno agli anni Settanta/ Sessanta del I sec. a.C. la creazione dell'intero organismo architettonico, che, nell'aspetto pervenutoci, è il risultato di molteplici modifiche condotte su un iniziale impianto in opera incerta di calcare molto accurata, che lo pone in sensibile anticipo rispetto ad altri edifici del Foro, in prevalente opera reticolata, e lo inserisce tra i teatri più antichi del Lazio ${ }^{12}$.

8 Gli scavi, condotti in più riprese, hanno restituito finora notevoli strutture ${ }^{13} \mathrm{e}$ materiali di rilevante interesse anche in rapporto alle variazioni urbanistiche collegate alla trasformazione del Foro in forme monumentali.

Del complesso sono stati accertati i limiti: a est, il poderoso muraglione in opera incerta (vd. supra n. 11); a ovest, un muro curvilineo pertinente all'emiciclo teatrale. Quest'ultimo si attesta ai resti di un asse viario basolato (via di Porta Nuova), identificabile con un diverticolo del decumano (via Anita Garibaldi), che, sulla direttrice nord-sud, conduceva direttamente all'ingresso occidentale. 
10 del secolo scorso, il muraglione è comune a due ambienti distinti ma intercomunicanti: il più arretrato (B), la cd. «basilica», è da identificare con il parascaenium orientale; l'altro (L), definito dal muraglione stesso e da un colonnato antistante di cui rimangono gli alloggi per le basi, appartiene al braccio orientale della porticus post scaenam. Molto vario quanto a soluzioni planimetriche, stando alle fonti antiche (Vitr., 5, 9, 1-9) e alle evidenze archeologiche, questo spazio annesso era di solito abbellito da fontane, opere d'arte, effigi di personaggi illustri e poteva comprendere piccoli sacelli, edicole e monumenti celebrativi. La porticus di Terracina (dimensioni stimate circa $51,50 \times 22,50 \mathrm{~m}$ ), accessibile direttamente dal decumano, si articolava su quattro lati con ambulationes disposte attorno a una corte rettangolare. È verosimile che sul lato prospiciente la piazza essa presentasse una duplice fila di colonne, una sola invece sugli altri tre.

11 Oggi la struttura risulta poco leggibile: nel versante ovest, infatti, è del tutto obliterata dal denso sistema abitativo compreso tra via la Palma e via di Porta Nuova, mentre la parte rimanente è stata liberata da un soprastante caseggiato solo di recente. Dell'imponente quadriportico restano, in sostanza, i due tratti del muro di fondo dei bracci est e nord scanditi inizialmente da semicolonne a fasce alternate di opera incerta di calcare e di laterizi, in seguito sostituite da paraste con basi ioniche e capitelli marmorei. Si conservano inoltre alcune basi attiche e fusti di colonne rudentate (h. max. $2 \mathrm{~m}$ ), nonché resti della pavimentazione in lastre di bardiglio e Carrara, più volte rinnovate e rappezzate già in antico. L'importanza della costruzione è sottolineata dal pregio dei rivestimenti e degli elementi architettonici marmorei, come dimostrano, in particolare, le raffinate colonne della fronte prospiciente il foro, di un tipo finora attestato, nella stessa Roma, sempre in contesti di alto livello di inoltrata età imperiale (fine I-II sec./età severiana) ${ }^{14}$.

Del teatro in senso stretto finora sono stati portati in luce i due corridoi principali di accesso (aditus maximi) coperti da volta a botte (quello orientale a forma di L e quello occidentale, rettilineo), simmetrici ma non uguali a causa dell'orografia del terreno (fig. 3). Entrambi, allo sbocco nel catino teatrale, presentavano al di sopra dell'arcata il tribunal (si conserva quello orientale): questa sorta di palco sopraelevato, qui articolato in tre gradoni su cui erano sistemati i sedili per le persone di riguardo, era delimitato da parapetti lapidei, come attestano evidenti segni di lavorazione e fori di grappe per l'incasso nel rivestimento del ripiano inferiore. 
Fig. 3 - La cavea, settore orientale.

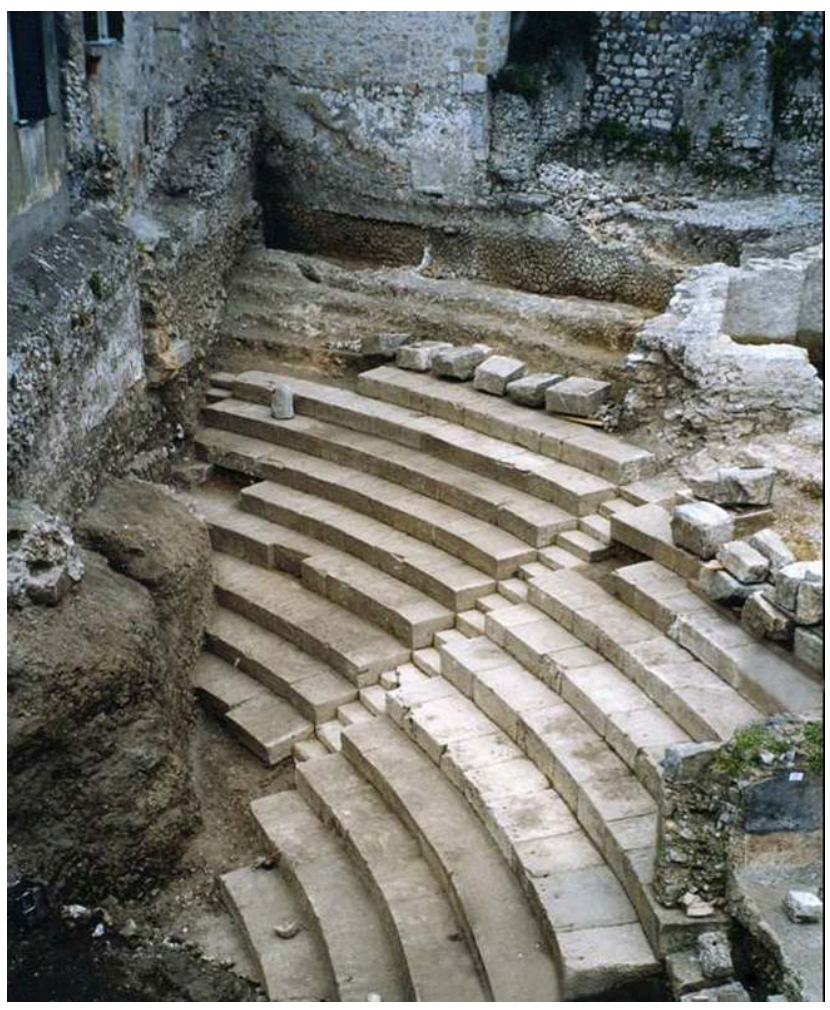

Foto N. Cassieri.

13 L'indagine dell'area è stata resa difficoltosa da una fitta rete di sottoservizi e dalle fondazioni di edifici moderni allo stato di rudere, da scantinati e ambienti sotterranei che in alcuni casi hanno raggiunto i livelli antichi. La quota dell'orchestra è risultata a $5 \mathrm{~m}$ di profondità dall'iniziale piano di campagna. Di questo spazio semicircolare è stato possibile indagare solo una porzione $(10,40 \times 2,75 \mathrm{~m})$, pavimentata in lastre di bardiglio e portasanta. Della cavea sono tornati in luce i primi due cunei a partire da est, composti, dietro a due gradoni per i posti d'onore (proedria), da 14 file di gradini messi in opera sul banco roccioso appositamente intagliato, appartenenti a due ordini di posti (ima e media cavea), raggiungibili da tre scalette radiali (scalaria), mentre due corridoi anulari di distribuzione scoperti (praecinctiones) la percorrono in senso orizzontale (fig. 4 $)^{15}$. Purtroppo la sommità della gradinata è andata perduta nella realizzazione di una cisterna, già alla fine del '700, e di un soprastante acciottolato stradale.

La prima praecinctio divideva i comodi sedili (bisellia) riservati alle alte cariche cittadine (magistrati, sacerdoti, decurioni) e a eventuali ospiti di rango, dalla ima cavea costituita da 5 file di gradoni destinati ai cavalieri: i numerosi fori da grappe in piombo visibili nei blocchi indicano chiaramente la presenza di un balteus di divisione dai sedili soprastanti; la seconda praecinctio si sviluppava all'altezza dei tribunalia, tra la ima cavea e la media cavea, quest'ultima composta da 9 file di gradini ( 6 ancora in buono stato): anche in questo caso, i segni superstiti lasciano supporre parapetti divisori in lastre di pietra. L'ordine mediano si concludeva con un corridoio anulare, coperto a volta (crypta) e provvisto di aperture, molto poco conservato; presumibilmente era preceduto da un analogo corridoio a cielo aperto. Al momento, della summa cavea non rimane alcuna traccia: tuttavia, una serie di indizi di ordine tecnico-costruttivo ne fanno 
presumere l'esistenza, probabilmente non prevista nel progetto iniziale, ma aggiunta in un momento successivo.

Fig. 4 - Planimetria del complesso teatro-portico, aggiornata al 2008/2009.

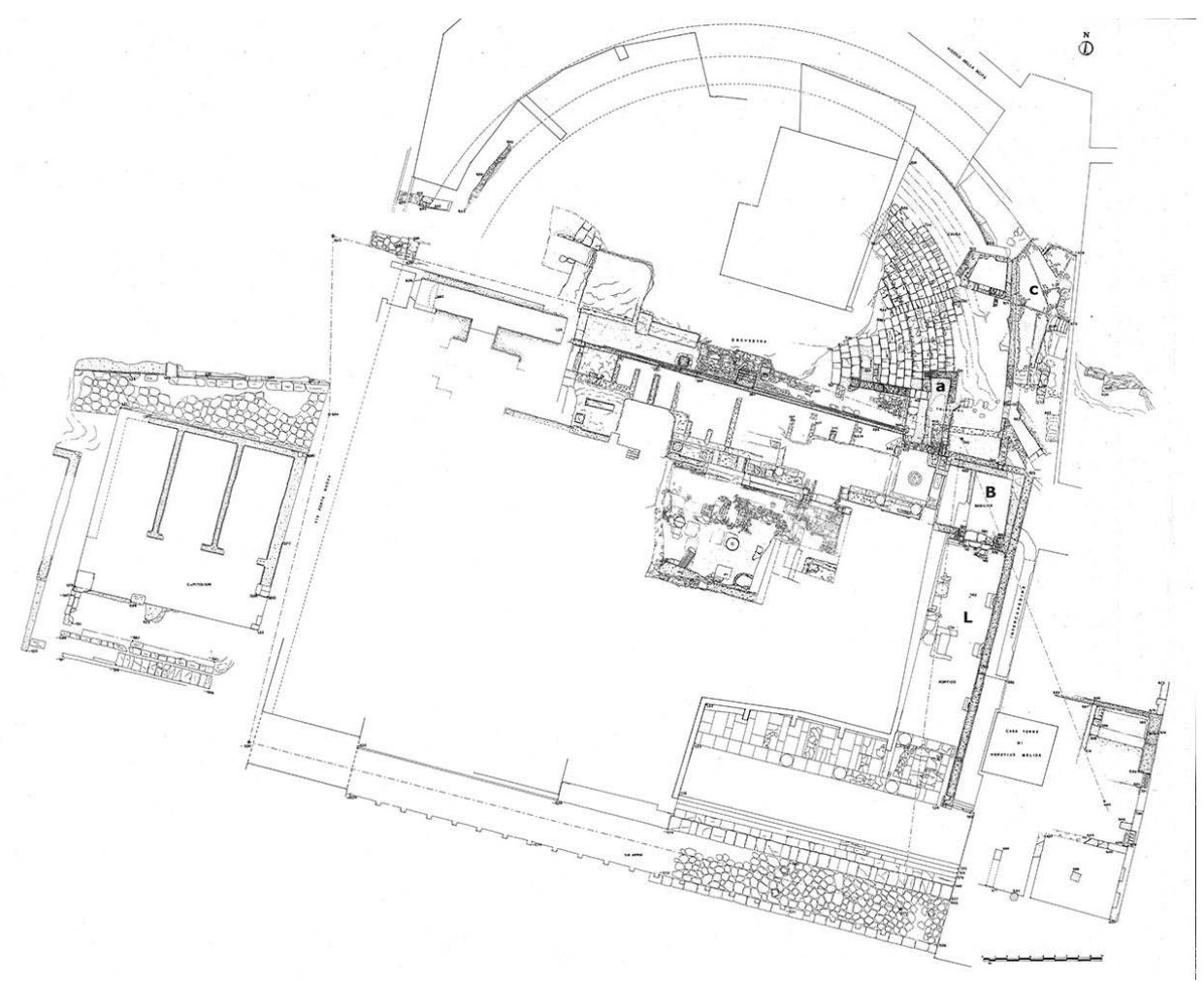

Foto N. Cassieri.

Con un gradino va appunto identificato un blocco rettangolare di calcare (lungh. $110 \mathrm{~cm} ; \mathrm{h} .30 \mathrm{circa}$ ), con indicazione di tre loca: esso infatti reca incisi sulla fronte, entro tre spazi contigui distinti da linee verticali che fissavano grosso modo l'ampiezza della seduta, $\mathrm{i}$ nomi di altrettanti fruitori dei posti ${ }^{16}$. I personaggi sono menzionati in maniera diversa: in alto a sinistra, nel primo campo, su un piano leggermente ribassato resta il gentilizio AEBVTIVS, in caso genitivo, ben attestato nel Lazio e in Campania. La parte sottostante invece appare scalpellata: ciò non stupisce considerato che, scaduto il diritto del locus, il nome veniva cancellato per far posto a un nuovo assegnatario. Al centro, il titolare è individuato solo da due lettere C. R., riferibili a elementi onomastici non identificabili. Per le dimensioni ridotte rispetto a quelle laterali è verosimile che questo locus sia stato ricavato in un secondo momento. A destra, l'iscrizione tracciata su due righe restituisce, nella parte superiore, il nome di una donna, FVFIA, indicata con il gentilizio al nominativo, già noto a Terracina ${ }^{17}$; nella zona sottostante, la cifra VIII specificava, solo in questo caso, il numero del posto a sedere nell'ambito del pertinente tratto di gradinata. Nell'insieme le caratteristiche paleografiche orientano per una datazione ancora in età tardo-repubblicana. Il gradino potrebbe provenire da un punto imprecisato della media cavea, quando la distinzione di genere prevista dalla rigida legislazione augustea sulla distribuzione del pubblico a teatro (Lex Iulia theatralis) non era stata ancora introdotta.

Nel quadrante occidentale della cavea, invece, un piccolo saggio del 2004 ha permesso di riportare alla luce cinque sedili ancora in sito: essi costituiscono un confortante 
indizio circa la sopravvivenza delle gradinate che, su questo lato (ancora da indagare), erano probabilmente addossate a una sostruzione artificiale, come sembra suggerire il ritrovamento in prossimità dell'aditus di un tratto di muro semicircolare incassato nel suolo, riconducibile a presumibili opere di sostegno.

Quanto alla costruzione scenica $(36,80 \times 8,20 \mathrm{~m})$, si tratta del settore che più degli altri è stato interessato da una serie di trasformazioni in nome di una maggiore funzionalità $\mathrm{e}$ di una progressiva nobilitazione. Oggi la scaenae frons si presenta costituita da un alto podio ( $\mathrm{h} .: 1,40 \mathrm{~m}$ ) formato da tre avancorpi in conglomerato cementizio rivestiti di lastre di travertino con cornici modanate alla base e alla sommità, sul quale poggiano alcune basi in marmo pertinenti al colonnato che si sviluppava su due ordini: quello inferiore, costituito da grandi fusti monolitici di bardiglio (h.: $4,80 \mathrm{~m})^{18}$, sormontati da capitelli ionici in marmo bianco come le basi; quello superiore, invece, composto forse da colonne più piccole in marmo africano, probabilmente munite di capitelli corinzi (fig. 5). In corrispondenza delle colonne la parete di fondo è scandita da paraste un tempo rivestite di marmo e provviste di basi e capitelli; realizzata in opera incerta e opera reticolata che si alternano senza un criterio apparente, essa si addossa al preesistente muro di fondo in incertum del braccio settentrionale della porticus. Nel frontescena di tipo rettilineo sono state indagate due delle tre aperture canoniche, quella centrale (valva regia) e quella orientale (porta hospitalis). Il tavolato ligneo del pulpitum era sostenuto da numerosi setti murari non tutti coevi, e da una frons anch'essa rettilinea (restano tracce della primitiva orchestra sotto la frons pulpiti).

Fig. 5 - Porzione dell'edificiob scenico (scavi 2005/2007).

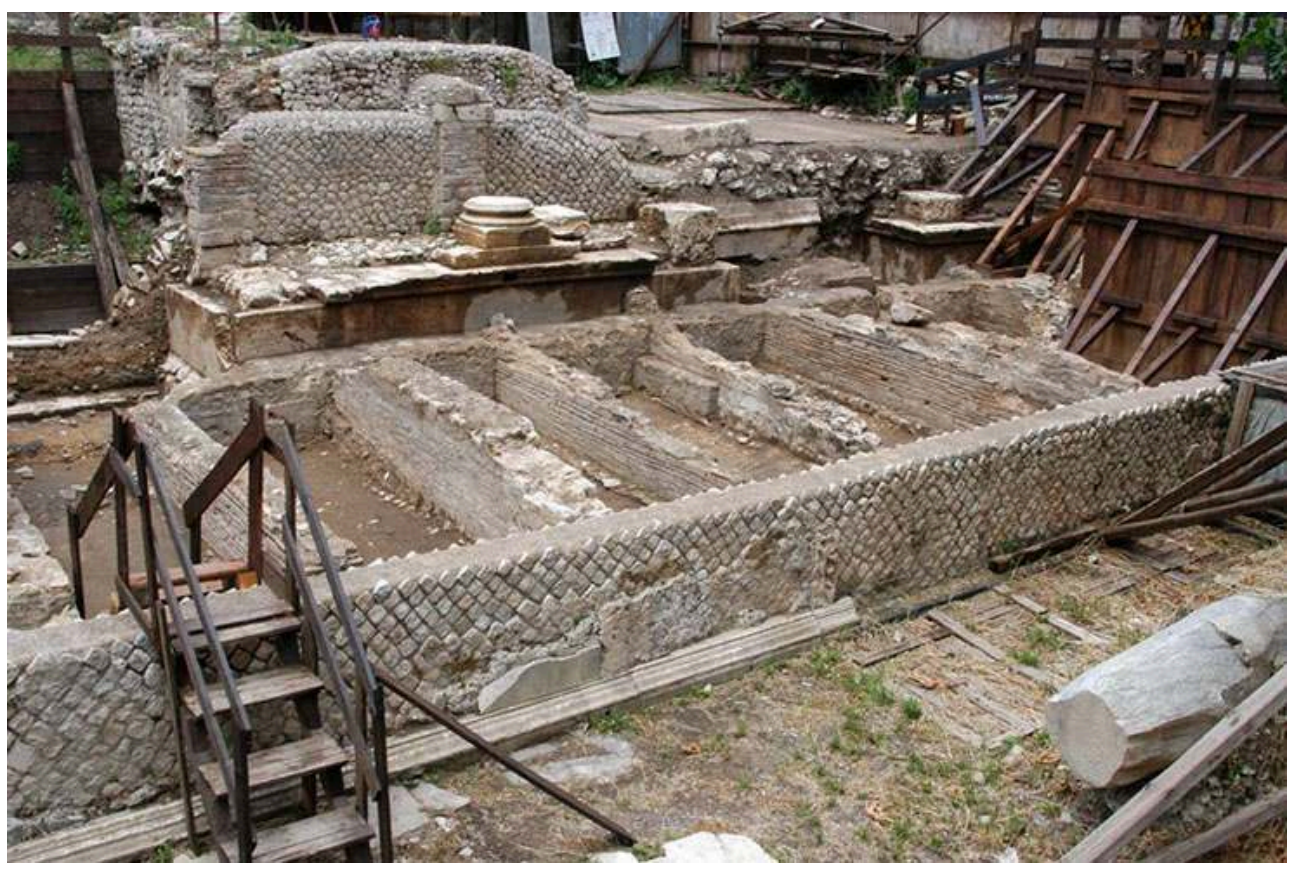

Foto N. Cassieri.

In estrema sintesi, va rilevato che, nell'impianto originario, la situazione si presentava del tutto diversa dal momento che orchestra, scena e porticus retrostante giacevano alla medesima quota e che solo in un secondo tempo si procedette alla creazione del podio, il quale, a vista nel primo periodo, venne poi nascosto agli spettatori da un pulpitum rialzato. 
19 Soltanto con la fase di piena monumentalizzazione, la terza, si raggiunse l'assetto definitivo che sostanzialmente ci è pervenuto. La radicale ristrutturazione del complesso, con cospicue modifiche nella planimetria e nei volumi, riguardò poi anche l'aspetto formale, con l'adozione di aggiornati sistemi decorativi e materiali ricercati e il potenziamento delle strutture dal punto di vista tecnico e funzionale, quali l'apprestamento dei meccanismi per la movimentazione del sipario.

20 Sotto il profilo cronologico, in base alle odierne conoscenze, è possibile individuare nel monumento almeno quattro fasi:

21 - Un impianto iniziale (scarsamente conservato) della prima metà del I sec. a.C., che si caratterizzava per i volumi semplici ed essenziali e l'uso diffuso di calcare locale per gli elementi architettonici e i piani pavimentali (lastre nell'orchestra e mosaici «a stuoia» con tessere oblunghe abbinate nell'ambulacro est della porticus).

22 - Una seconda fase, tra la metà del I sec. a.C. e l'età augustea, che vide mutamenti significativi nell'edificio scenico, con la costruzione del podio seppure ancora non completamente strutturato. È probabile che il frontescena si presentasse decorato con coppie di colonne disposte semplicemente su un unico ordine a inquadrare le tre aperture. Si può altresì ritenere che solo a questo punto sia stata definita l'articolazione architettonica della cavea nei suoi diversi settori.

23 - Una terza fase di rinnovamento e nobilitazione del teatro mediante una serie di interventi costruttivi inerenti soprattutto all'edificio della scena e la sua ornamentazione, può risalire all'età augustea, con il completamento di alcune parti ancora nel primo periodo giulio-claudio. Marmi pregiati di importazione presero allora il posto del modesto calcare nelle membrature architettoniche e nei rivestimenti parietali; costose lastre colorate sostituirono il sobrio mosaico bianco. Si inquadra in questa fase il raddoppiamento del muro di fondo della frons scaenae ai fini della sua sopraelevazione, eseguito in opera reticolata e incerta utilizzate in contemporanea. In concomitanza con la probabile realizzazione della summa cavea, si aggiunsero ulteriori colonne sul podio e un secondo ordine superiore. Inoltre fu rialzato di 1,50 $\mathrm{m}$ il livello del pulpitum, che venne raccordato all'orchestra tramite una scaletta posta al centro del proscaenium: la creazione del muro frontale in opera reticolata rivestita di marmo (lungh. max 29,50 m), conforme per altezza (5 piedi) ai precetti vitruviani, segna un diverso piano di spiccato del frontescena visto che la nuova sistemazione va a inglobare il podio fino alla cornice superiore. Alla base del muro, sul lato interno, si conservano alcuni dei pozzetti della fossa iposcenica che dovevano ospitare i pali lignei del sipario in posizione di riposo ${ }^{19}$. Il funzionamento dell'auleum era assicurato da efficienti meccanismi installati in due camere di manovra identificate sul fianco orientale della scena. Ai suddetti interventi si collegano anche la chiusura di un passaggio arcuato che affiancava l'ingresso principale al parascaenium orientale (B) e la riorganizzazione dello spazio circostante, stabilendo nuovi rapporti funzionali tra le varie parti dell'edificio. In particolare si ascrive a questo ambito la rettifica del vano $\mathrm{B}$, finalizzata alla copertura con una volta a padiglione decorata a motivi geometrici (fig. 6) e alla sua sopraelevazione mediante, murature caratterizzate anche qui dalla disinvolta compresenza di opera incerta e opera reticolata, in stretta relazione all'innalzamento del frontescena. 
Fig. 6 - Vano B, porzione di soffitto dipinto a fondo nero, ricomposta da minuti frammenti. 35-45 d.C.

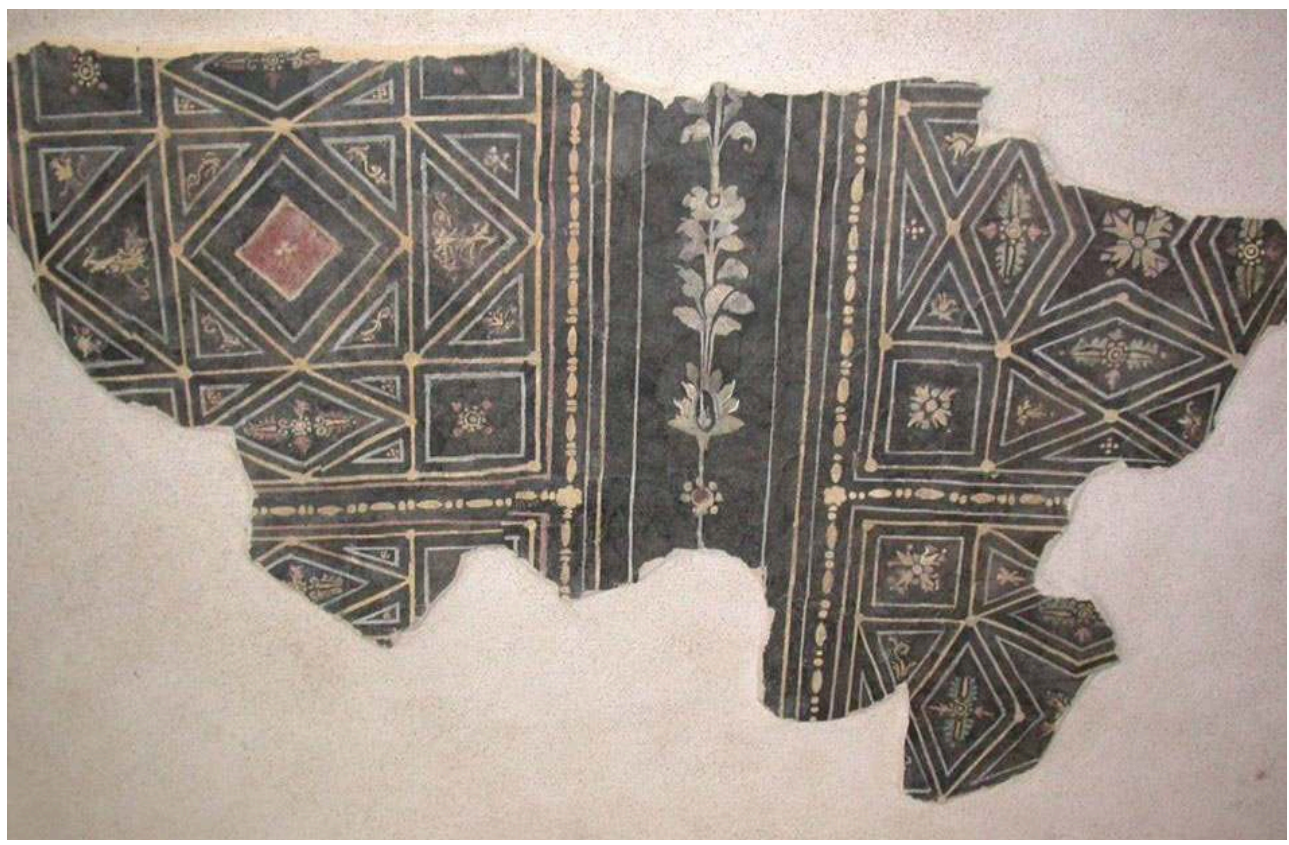

Foto N. Cassieri.

- Una quarta fase è da ricondurre a non prima dell'età dei Severi (fine II-primi decenni III sec. d.C.), che a Terracina si traduce in un periodo di particolare vitalità edilizia documentata da restauri e nuove opere soprattutto nella città «bassa» ${ }^{20}$. Nei decenni successivi si provvide piuttosto alla riparazione e al ripristino funzionale degli edifici, sovente con materiali di recupero: nel teatro, un reimpiego è evidente, per esempio, nella sistemazione del prospetto del pulpitum, dove porzioni di cornici vennero a integrare la modanatura di base e almeno quattro lastre iscritte, o parti di esse, messe in opera al rovescio, lo rivestirono per un tratto: la loro scomparsa ha lasciato nella malta di allettamento le impronte «in negativo» di resti di tituli, in corso di studio. Meno chiari sotto l'aspetto cronologico sono alcuni interventi di epoca tarda eseguiti nel momento della dismissione del teatro in quanto tale (es. vasche in cocciopesto e ambienti di dubbia natura, sulla summa cavea).

Nello scavo del catino teatrale, effettuato negli anni 2005-2007, i ritrovamenti più significativi si sono verificati nella fascia compresa tra il podio e l'orchestra dove, quasi a contatto col piano di calpestio antico, un alto strato di terra scura con vistose tracce di bruciato e crollo, presente in maniera costante nelle limitate aree indagate, ha restituito sculture, frammenti di epigrafi e di manufatti marmorei, che non è escluso fossero stati qui accumulati nell'ambito di un cantiere di spoglio dell'edificio antico. Nei livelli inferiori di questo strato frammenti di ceramica sigillata africana D (V-VI secolo) sembrano fornire indizi cronologici circa la definitiva defunzionalizzazione dell'edificio teatrale.

Tra i numerosi materiali merita di essere qui segnalata una monumentale statua di barbaro "Orientale $»^{21}$ (fig. 7), rinvenuta in tre grossi frammenti in prossimità della frons scaenae. Ricavata da due blocchi di giallo antico, doveva avere le parti scoperte (volto e mani), non conservate, in marmo bianco. La figura, gravitante sulla gamba destra, ha il braccio corrispondente flesso e levato in alto; il sinistro, staccatosi di netto all'attacco 
della spalla e al momento mancante, era verosimilmente abbassato. Vestito nel caratteristico costume variopinto (brache, tunica, mantello, copricapo frigio), il personaggio si addossa a un pilastro che ne specifica la funzione di telamone. La scultura rientra tipologicamente nel gruppo di barbari «Orientali», eretti o inginocchiati in atteggiamento di sostegno, che, nell'età augustea, vennero collocati in importanti contesti pubblici, quali la Basilica Emilia, dove una folta schiera di questi personaggi aveva il compito di esaltare la «vittoria» di Augusto sui Parti nel 20 a.C., celebrando, nel contempo, il potere e la supremazia di Roma ${ }^{22}$. La scultura è databile nell'ambito della prima metà del I sec. d.C. e mostra stringenti confronti con il noto esemplare già della collezione Mattei (Roma, Palazzo Altemps) ${ }^{23}$. Non si possiedono al momento dati sufficienti sulla collocazione originaria: è possibile che, insieme a una seconda statua, testimoniata finora da un avambraccio e dalla terminazione di un berretto a punta, facesse parte della decorazione del frontescena, oppure della porticus retrostante.

Fig. 7 - Statua di «barbaro» orientale, in giallo antico, dopo il restauro (ex Museo Civico Pio Capponi).

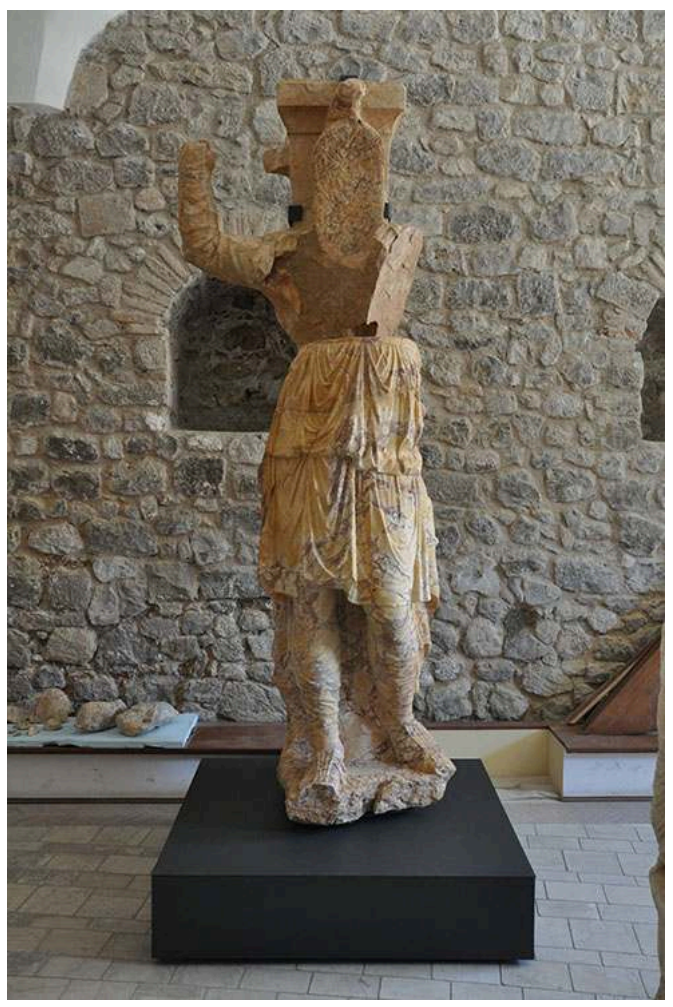

Foto N. Cassieri.

Accostato al muro del pulpitum è stato rinvenuto nel 2007 un altare parallelepipedo (h. $1 \mathrm{~m}$ circa) in marmo, al momento nei depositi comunali; sui lati sono rappresentati gli oggetti rituali del sacrificium, mentre sul retro è un albero di alloro fiancheggiato da due cigni, che riconduce al simbolismo di Apollo traslato in quello di Augusto e della sua dinastia. Sulla fronte è incisa la dedica IOVI AXVRI / SACRVM (fig. 8), di particolare interesse in quanto rappresenta la prima attestazione epigrafica certa del culto di Giove Axur/Anxur/Axurus a Tarracina ${ }^{24}$, oltre che del culto medesimo nell'ambito dell'edificio teatrale. La tipologia del manufatto, i motivi decorativi e le caratteristiche paleografiche del testo indicano una datazione nel I sec. d.C. Qualora la sua posizione 
non sia quella originaria, si deve presumere l'esistenza di un piccolo sacello (o di una semplice edicola) dedicato al dio, all'interno del complesso monumentale ${ }^{25}$.

Fig. 8 - Ara con dedica a luppiter Axur, rinvenuta accostata alla frons pulpiti.

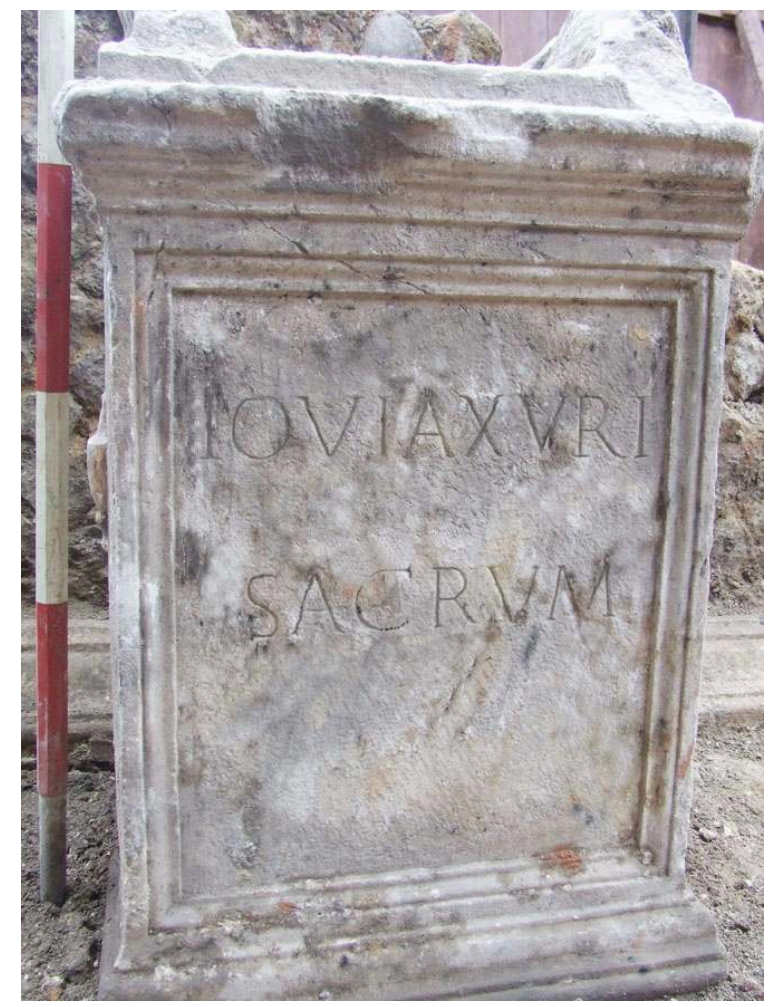

Foto N. Cassieri.

Tra le acquisizioni che meritano speciale attenzione figura soprattutto l'iscrizione incisa su una spessa lastra di marmo bianco, rinvenuta nello strato di riempimento sopra menzionato, fratta in numerosi frammenti contigui, che permettono di ricomporre quasi per intero il supporto originario $(21,6 \times 77,1 \times 3,8 \mathrm{~cm})$; il testo, delimitato da cornice modanata formata da listello e gola rovescia, è mutilo in basso a sinistra e soprattutto a destra. Le lettere presentano un bel modulo quadrato, con larghe graffie alle estremità dei tratti $(\mathrm{h} .:$ 4,5-3,1 cm); una qualche influenza della scrittura actuaria si osserva nelle $\mathrm{T}$, il cui tratto orizzontale ha andamento svolazzante (rr. 2, 3); i segni di interpunzione sono di forma triangolare, usati in maniera regolare; almeno una lettera è montante ( $\mathrm{T}$ di $\mathrm{r} .3$ ); il retro è lavorato a scalpello, tranne che lungo i margini superiore e inferiore dove si è fatto ricorso alla gradina. Si conserva nei depositi comunali (Inv. SAL153365 [2016]).

\section{La nuova iscrizione del triumviro Lepido}

\section{Gian Luca Gregori}

Dalla ricomposizione dei sette frammenti iscritti possiamo ricostruire il seguente testo (fig. 9): 
M(arcus) Aimilius M(arci) f(ilius) Lepid.[us] / pontifex maxumus, cọ(n)[s(ul) II], / III uir r. rei) [p(ublicae) c(onstituendae) i]ter(um), mag(ister) eq(uitum) III, pr(aetor), imp(erator) III, triu[ [mp(hauit) II?]. abbreviata deaspirata del verbo, in considerazione della non troppo ampia lacuna di destra; essa trova del resto confronti nei Fasti triumphales e nell'iscrizione sul Mausoleo di L. Munatius Plancus ${ }^{26}$.

Fig. 9 - Iscrizione del triumviro M. Emilio Lepido, dopo la ricomposizione.

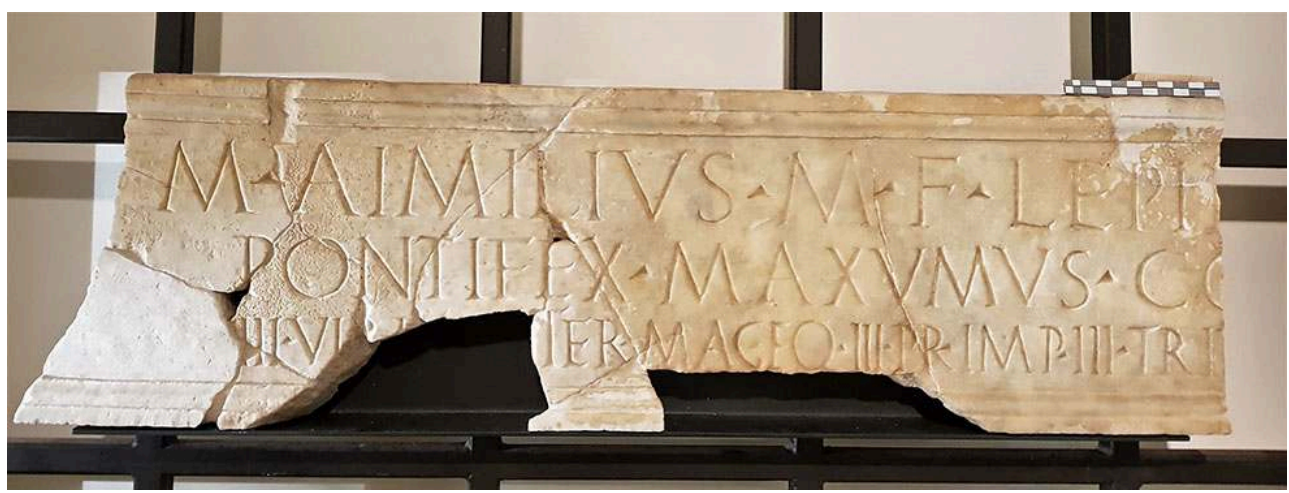

Foto G. de Simone.

L'eccezionalità dell'iscrizione sta nel fatto che si tratta di uno dei pochissimi testi sopravvissuti che ricordi il triumviro Lepido, a prescindere dalla sua presenza nei Fasti trionfali ${ }^{27}$ e consolari, e dalla sua ben poco lusinghiera menzione nel cosiddetto elogio di Turia ${ }^{28}$. Rimanendo per ora a livello di ipotesi l'attribuzione a Lepido di una dedica da Emporiae, nell'Hispania Citerior, dove il personaggio è onorato come patrono ${ }^{29}$, finora era con certezza riferibile al triumviro solo la dedica posta per decreto dei decurioni di Thabraca, in Africa Proconsolare ${ }^{30}$ :

M(arco) Lepido imp(eratori) / tert(ium), pont(ifici) max(imo), / IIIuir(o) $r($ ei) p(ublicae) c(onstituendae) bis, co(n)s(uli) / iter(um), patrono / exd(ecreto) d(ecurionum).

Nessuno dei due testi sopravvissuti fa riferimento agli incarichi proconsolari ricoperti da Lepido, prima nella Spagna Citeriore (48-47 a.C.), poi contemporaneamente nella Gallia Narbonense e nella Spagna Citeriore (44-42 a.C.), infine in Africa (40-36 a.C.) $)^{31}$. Il testo africano omette anche le funzioni di magister equitum e di pretore, mentre pone l'accento sulle tre acclamazioni imperatorie.

Il testo terracinese invece relega gli onori di carattere militare (acclamazioni e trionfi) all'ultimo posto, ma conferma, al pari del testo africano, che nel 37 a.C. anche a Lepido erano stati rinnovati i poteri triumvirali. ${ }^{32} \mathrm{Mi}$ pare che l'enfasi cada qui soprattutto sulle cariche religiose e politiche, secondo una successione che vede al primo posto, con lettere d'altezza maggiore, il pontificato massimo, cui egli era stato eletto dopo la morte di Cesare ${ }^{33}$, seguito dai due consolati (il primo nel 46 a.C., con lo stesso Cesare, il secondo nel 42 a.C., con L. Munatius Plancus). Seguono in ordine discendente le magistrature straordinarie: il triumvirato nella sua definizione ufficiale, iterato (43-38; 37-36 a.C.) e la funzione di magister equitum, ricoperta durante la dittatura di Cesare (45-44 a.C.). Viene a questo punto la pretura (49 a.C.), assente nel testo africano e la cui menzione potrebbe sembrare fuori luogo alla luce delle successive e ben più importanti 
cariche, se non fosse che fu proprio durante la pretura che Lepido propose l'attribuzione della dittatura a Cesare, allora impegnato in Spagna ${ }^{34}$.

Nel complesso sembra a me innegabile il forte valore di autorappresentazione del nuovo testo a favore di Lepido: attraverso la ricerca del consenso municipale egli sperava forse di elevare la sua figura al livello di Ottaviano e Antonio, entrambi con tre salutazioni imperatorie all'attivo ${ }^{35}$. Per questo vengono forse enfatizzate nel nuovo documento quelle cariche che lo avevano posto in stretta relazione con Cesare, il quale, a suo tempo aveva preferito lui ad altri per la delicata carica di magister equitum. La realtà dei fatti era però molto diversa e si assisteva, almeno dal 42 a.C., a una progressiva emarginazione di Lepido.

L'iscrizione di Terracina, al pari del testo africano, dovrebbe avere come terminus ante quem il settembre del 36 a.C.: dopo la vittoria a Nauloco (3 settembre) infatti e la successiva occupazione di Messina, le legioni di Lepido, cui si erano unite quelle di Sesto Pompeo passate dalla sua parte, furono indotte ad abbandonare il loro comandante a favore di Ottaviano, come recitano i Fasti Cumani: [... exer]citus Lepidi tradidit se Caesari ${ }^{36}$.

Ottaviano seppe cogliere al volo quell'occasione per togliere a Lepido, del cui supporto egli non aveva ormai più bisogno, ogni potere e carica, con l'eccezione del pontificato massimo e «imponendogli» una relegatio nella sua villa di Circeii, salvo richiamarlo a Roma ogni volta che ritenesse opportuna la sua presenza in Senato o l'espletamento del suo ruolo di pontefice massimo ${ }^{37}$.

Il terminus post quem sarà rappresentato invece dall'autunno del 37 a.C., quando a Taranto Ottaviano e Antonio (assente Lepido) decisero il rinnovo dei poteri triumvirali per un altro quinquennio ${ }^{38}$.

Mentre però la dedica africana non desta particolare scalpore, provenendo da una provincia che era in quel momento controllata proprio da Lepido (anche se sul suo operato di quegli anni in quella provincia le fonti, evidentemente di parte, tacciono) ${ }^{39}$, il nuovo testo viene dall'Italia, che allora era sotto il controllo di Ottaviano. Se ne può trovare forse una buona motivazione nelle strette relazioni clientelari che da tempo dovevano legare gli Aemilii Lepidi a Terracina; essi, del resto, oltre alla vicina villa di Circeii, fin dagli inizi del II sec. a.C. avevano, come s'è detto, interessi economici nell'agro terracinese $e^{40}$, al pari di altri esponenti della nobiltà senatoria romana. Dovremmo in ogni caso dedurre che prima della spedizione in Sicilia non si prevedesse l'ormai imminente allontanamento di Lepido dalla scena politica.

Quanto poi alla carica di magister equitum l'iscrizione di Terracina sembra ora destinata ad alimentare la discussione apertasi tra gli studiosi dopo la recentissima pubblicazione dei Fasti Privernates ${ }^{41}$, dove a proposito dell'anno 44 a.C. si legge:

C(aius) Iulius Caesar IV dict(ator) abdic(auit) ut perpet(uo?) [---] / M(arcus) Aemilius Lepid(us) II mag(ister) eq(uitum) abd(icauit) ut perpet(uo?) [---] / quoad dict(ator) Caesar esset ...

41 Nei primi mesi del 44 (tra il 9 e il 15 febbraio) e in concomitanza con l'abdicazione di Cesare dalla sua quarta dittatura e l'assunzione della dittatura perpetua, anche Lepido avrebbe dunque abdicato, per assumere in perpetuo la carica di magister equitum, carica che quindi si prevedeva che egli avrebbe conservato anche dopo la sua partenza, prevista per quello stesso anno, verso le province a lui destinate di Gallia Narbonense e Hispania Citerior; vista la sua assenza da Roma, egli sarebbe stato affiancato nel ruolo, secondo un modello privo di precedenti, di volta in volta da altri magistri equitum per il 
controllo dell'Urbe: nel 44 a.C. M. Valerius Messalla, nel 43 a.C. Cn. Domitius Calvinus, mentre, secondo l'ipotesi di F. Càssola, nel 42 a.C. sarebbe dovuto finalmente toccare al nipote C. Octavius ${ }^{42}$.

Tutto però saltò, perché, morto Cesare, la dittatura fu abolita, il potere tornò momentaneamente nelle mani del console Antonio, Lepido perse le sue funzioni e nessuno dei magistri equitum designati da Cesare entrò mai in carica.

Altri Fasti conservano il riferimento a quegli anni, ma tutti sono purtroppo mutili proprio in corrispondenza della carica di magister equitum di Lepido ${ }^{43}$. Sulla scia dell'edizione dei Fasti Capitolini di A. Degrassi, finora si era sempre integrato il numerale III in relazione alla funzione svolta nel 44 a.C. da Lepido ${ }^{44}$. I Fasti Privernates hanno sollevato dubbi sulla legittimità di tale integrazione e l'ipotesi di un errore di incisione del lapicida (che avrebbe reinciso quanto in realtà era riferito a Cesare), avanzata da qualcuno, non ha convinto il primo editore ${ }^{45}$. Il testo terracinese sembra ora fornire un elemento a favore della interpretazione tradizionale, che, indipendentemente dalla nuova iscrizione, è stata accolta anche nell'integrazione dei recentissimi Fasti Albenses ${ }^{46}$.

In sostanza non vi fu nel 44 a.C. la nomina di Lepido a magister equitum in perpetuo, ma III, oppure, se vi fu, essa di fatto non ebbe seguito a causa dell'assassinio di Cesare, per cui a distanza di qualche anno si poteva semplicemente parlare di una carica ricoperta per la terza volta (e per di più solo per pochi mesi).

Anche la terza acclamazione imperatoria di Lepido, finora attestata solo dalla dedica di Thabraca, viene ora definitivamente confermata dalla nuova iscrizione, che, come ora si vedrà, termina proprio con la menzione delle tre acclamazioni e probabilmente dei due trionfi di Lepido.

\section{La troisième acclamation impératoriale de Lépide}

Jean-Baptiste Refalo-Bistagne

L'inscription de Terracine se clôt par deux éléments de nature politico-militaire : les acclamations impératoriales de Lépide et ses triomphes. Comme évoqué plus haut, si l' imp(erator) III est facile à interpréter, les lettres tri[- - - ] sont d'intégration incertaine. Néanmoins, il est fait probablement référence, en l'occurrence, aux triomphes de celui qui fut trois fois magister equitum : alors qu'il est d'usage que les acclamations et les triomphes soient de nature militaire ${ }^{47}$, ceux de Lépide ne sont pas gagnés sur le champ de bataille mais résultent de ses qualités de négociateur. La découverte de l'inscription de Terracine pose de nouveaux problèmes concernant Lépide, notamment à propos de ses acclamations impératoriales. Les deux premières sont assez classiques dans le contexte des années 50 av. J.-C. ${ }^{48}$ En 48-47, Lépide est proconsul d'Espagne Citérieure. Son voisin propréteur d'Espagne Ultérieure, Q. Cassius Longinus ${ }^{49}$, menacé par une mutinerie initiée par son questeur, fait appel à Lépide afin de conserver la paix dans sa province. L'intervention de ce dernier n'est pas militaire mais diplomatique : la tenue de négociations entre le questeur M. Claudius Marcellus Aeserninus et le propréteur Longinus aboutit à un retour à la paix ${ }^{50}$. En récompense de cet accomplissement, Lépide obtient de César un triomphe célébré à la fin de l'année 47, le consulat pour 46 et la 
charge de magister equitum ${ }^{51}$. Il est hautement probable qu'à ces honneurs soit aussi ajoutée une première acclamation impératoriale.

La seconde est également obtenue en Espagne, lors du second proconsulat en Citérieure de Lépide en 44. Le contexte est néanmoins très différent : après la mort de César, Sextus Pompée s'affirme comme un protagoniste mettant à profit l'héritage de son père dans la péninsule ibérique ${ }^{52}$. Afin d'empêcher un conflit, le sénat envoie ${ }^{53}$ le nouveau grand Pontife ${ }^{54}$ négocier la paix avec le fils du Grand Pompée. Une fois sa mission accomplie, l'assemblée des Pères le récompense par une supplication, une seconde acclamation impératoriale ${ }^{55}$, une statue équestre dorée sur les Rostres et un deuxième triomphe ${ }^{56}$. Avant la découverte de l'inscription de Terracine, seule l'inscription de Thabraca évoquait la troisième acclamation impératoriale de Lépide, qui est ainsi définitivement confirmée dans ce nouveau document. Cependant, s'il semble acquis qu'elle ait eu lieu en Afrique, son contexte et ses modalités restent incertains.

À Bologne, en septembre 43, Antoine, Octavien et Lépide se rencontrent et formalisent le Triumvirat. Au-delà de pouvoirs politiques exceptionnels, chacun des trois hommes obtient des provinces : l'Afrique, la Numidie, la Sardaigne et la Sicile pour Octavien; la Gaule, à l'exception de la Narbonnaise, pour Antoine et la Narbonnaise et toute l'Espagne pour Lépide ${ }^{57}$. La bataille de Philippes marque la fin du pouvoir réel du grand Pontife au sein du triumvirat. Alors que celui-ci est resté à Rome en qualité de consul, la victoire d'Antoine et Octavien contre Brutus et Cassius leur a donné une gloire et un pouvoir leur permettant d'ignorer les souhaits de Lépide ${ }^{58}$. Les deux césariens peuvent de fait marginaliser leur collègue : à leur force militaire conséquente s'ajoutent sept des dix légions de Lépide ${ }^{59}$. Par la suite, après la bataille de Philippes, les vainqueurs procèdent à une redistribution des provinces : l'Espagne et la Narbonnaise sont alors retirées à Lépide au bénéfice d'Octavien, sans aucune compensation. Sur cet épisode, les sources divergent: Dion Cassius ${ }^{60}$ évoque une mise en réserve, avec la possibilité de lui conférer l'Afrique et la Numidie - alors antoniennes - en cas de nécessité. Appien fait part d'une accusation de trahison envers Lépide ${ }^{61}$ : ses collègues lui reprocheraient d'avoir négocié avec Sextus pendant leur absence. Toutefois, l'historien alexandrin précise que si ce grief se révélait faux, alors l'Afrique et la Numidie passeraient d'Antoine au consul de 42. Ainsi, soit parce qu'il fut blanchi de ces attaques ${ }^{62}$, soit parce qu'Octavien souhaite s'assurer de son soutien dans la guerre de Pérouse face à L. Antonius et Fulvia ${ }^{63}$, l'Afrique est donnée à Lépide. En conséquence, en partant pour ses provinces à l'été 40 , le grand Pontife sert la politique du fils de César : ce dernier (re)trouve un allié ${ }^{64}$, lui permettant de même d'envoyer en Afrique six légions d'Antoine à la fidélité douteuse ${ }^{65}$. Plus tard, cette nomination est ratifiée lors de la Paix de Brindes, en 40, malgré l'absence de Lépide ${ }^{66}$, alors dans sa province.

En Afrique, la situation rencontrée par Lépide à son arrivée est épineuse. Tout d'abord, la province autour de Carthage - l'Africa - appartient encore officiellement à Antoine ${ }^{67}$. Sur place, un légat, T. Sextius, contrôle ainsi cet espace, mais domine aussi militairement la Numidie adjacente ${ }^{68}$. Selon les sources, à l'arrivée de Lépide, Sextius se soumet au grand Pontife sans hostilité. Dion Cassius évoque deux explications possibles à ce comportement: il estime soit que cette nomination de Lépide a reçu l'aval d'Antoine, soit que sa force n'était pas assez importante pour résister au triumvir dans un affrontement ${ }^{69}$. Par ailleurs, Sextius remet au consul de 42 les troupes qu'Antoine lui avait confiées pour sa campagne contre les Parthes. Ainsi, à la fin de l'année 39, lorsque le triumvir envoie Furnius ${ }^{70}$ en Afrique afin que Sextius lui restitue ces quatre légions, 
celles-ci sont déjà passées sous le commandement de Lépide ${ }^{71}$. De manière générale, les sources sont silencieuses au sujet du gouvernement du grand Pontife en Afrique ${ }^{72}$. En effet, leur narration est systématiquement focalisée sur Antoine, Octavien et même parfois Sextus Pompée ; Lépide n'est évoqué que lorsqu'il interagit avec les principaux protagonistes de la période. Ainsi, pour toute l'année 38, seul Dion Cassius évoque une action de Lépide, lorsque celui-ci refuse de répondre à une convocation d'Octavien ${ }^{73}$. Cet éloignement n'est pas que narratif: en 37, lors de leur réunion à Tarente prolongeant le Triumvirat pour cinq ans, Antoine et le jeune César ne souffrent pas la présence de Lépide. De plus, dans tous les récits des sources sur l'événement, Lépide n'est jamais mentionné ${ }^{74}$.

50 Avant la découverte de l'inscription de Terracine, le seul témoignage d'une troisième acclamation impératoriale de Lépide en Afrique $^{75}$ était une dédicace, retrouvée à Thabraca, à environ 150 kilomètres de Carthage ${ }^{76}$. Ce document confirme en premier lieu la participation continue du grand Pontife au triumvirat après les accords de Tarente, en dépit de son absence ${ }^{77}$. Par ailleurs, il établit que Lépide est le patron de la cité de Thabraca, après lui avoir certainement concédé quelque avantage ${ }^{78}$. Enfin l'inscription évoque une troisième acclamation impératoriale obtenue par Lépide. La dédicace est datée entre 37 - après la rencontre de Tarente, où Lépide devient triumvir $b i s^{79}$ - et juillet 36 , lorsque le grand Pontife embarque pour la Sicile. L'acclamation a donc dû avoir lieu entre 43 et 37-36. Nous éliminons une datation haute : après son triomphe de décembre 43 , le triumvir est consul en $42^{80}$, en charge des affaires courantes pendant qu'Antoine et Octavien combattent Brutus et Cassius en Orient. Cela écarte toute acclamation pour la durée de sa charge. De même, nous excluons l'année 41: même si Lépide est toujours en Italie durant la guerre de Pérouse, la perte de ses provinces après Philippes a réduit son prestige par rapport à celui de ses collègues ainsi que son champ d'action. Enfin, nous rejetons aussi les six premiers mois de l'année 40, où Lépide est toujours inactif - ou du moins, négocie avec Octavien pour obtenir une nouvelle province. Il nous semble donc que cette obscure acclamation n'a pu avoir lieu qu'en Afrique, posant le terminus post quem à l'été 40 .

51 L'hypothèse d'une erreur dans l'inscription est désormais irrecevable, la nouvelle dédicace de Terracine faisant également état de la troisième acclamation impératoriale de Lépide. Dans son compte rendu de la découverte de l'inscription africaine, J. Guey n'accorde que peu d'intérêt à l'acclamation ${ }^{81}$. J.-M. Roddaz ${ }^{82}$ émet l'hypothèse d'une acclamation à la fin de l'année 40, au moment où Antoine et Octavien sont eux-aussi proclamés imperatores : le grand Pontife aurait imité ses collègues sans mener d'actions militaires. Cette explication n'éclaire pas sur l'acte de Lépide par lequel l'acclamation est obtenue : hors de toute action militaire, cela pourrait être une négociation, comme ce fut le cas en 48 et en 44 . R. Weigel évoque trois pistes : un "incident» durant son administration, une rixe ${ }^{83}$ avec les troupes de Sextus Pompée ou une rébellion africaine ${ }^{84}$. La rixe avec les troupes de Sextus nous semble peu probable: aucun témoignage ne relate un accrochage entre le fils de Pompée et un des triumvirs avant la guerre de Sicile ${ }^{85}$.

L'hypothèse la plus plausible serait liée à la "transition pacifique » du pouvoir entre T. Sextius et Lépide, ce premier se rangeant à l'autorité du triumvir. En effet, Sextius détient dans sa province un nombre important de troupes et a éliminé tous ses adversaires. Les sources, pour justifier son attitude, évoquent un respect des triumvirs ${ }^{86}$. Toutefois, il est possible que Sextius, profitant de sa puissance, ait tenté de 
s'opposer à Lépide. Ceci pourrait alors expliquer la surprise d'Antoine en constatant que les quatre légions qu'il a confiées à son légat ont été reprises par Lépide ${ }^{87}$ : Sextius lui a-t-il vraiment cédé volontairement ses troupes ?88 De même, comme évoqué par J.M. Roddaz ${ }^{89}$, il semblerait opportun de dater l'acclamation dans les derniers mois de l'année 40. Cela montre tout d'abord une volonté de Lépide d'affirmer la continuité de sa participation au triumvirat - même éloigné en Afrique, alors qu'Antoine et Octavien sont aussi acclamés imperatores à cette époque. Ainsi, seule l'hypothèse d'un accrochage avec Sextius tend à confirmer cette datation.

Si l'inscription de Terracine confirme définitivement la troisième salutation impératoriale de Lépide, le manque de sources archéologiques et le peu d'informations apportées par les textes, réduisent, en l'absence d'autres éléments, les conditions de son octroi à l'obscurité.

\section{Conclusione}

Restano per ora sconosciute le ragioni per le quali venne collocato in età triumvirale un ricordo epigrafico del triumviro Lepido nel teatro di Terracina. Gli Aemilii Lepidi fin da età repubblicana risultano avere avuto interessi economici nell'agro della colonia $\mathrm{e}$ certamente possedevano una villa nella vicina Circeii. Tuttavia, vista la sua caduta in disgrazia nel 36 a.C. (che coinvolgerà di lì a poco anche i due figli, ma non i nipoti) ${ }^{90}$, pare inverosimile che potessero ancora convergere su Lepido, dopo il settembre di quell'anno, dediche, statue, patronati. Anche se non vi fu mai nei suoi confronti alcuna forma di damnatio (con relativa erasione del nome) ${ }^{91}$, Ottaviano non fece mai mistero della sua mal disposizione d'animo nei confronti dell'ex collega ${ }^{92}$ e dalle fonti non si evince vi sia stata una successiva riabilitazione della sua memoria tale da giustificare un onore a posteriori.

Non si può a priori escludere un intervento del triumviro nel potenziamento del teatro sotto l'aspetto strutturale, essendo documentata sul piano archeologico una fase significativa tra quella originaria nella prima metà del I sec. a.C. e l'età augustea. Tuttavia, in considerazione delle misure della lastra e dell'altezza dei caratteri, la provenienza dall'interno del teatro e l'uso del nominativo non autorizzano a pensare che si tratti della dedica dell'opera da parte del triumviro. L'impianto del testo rinvia piuttosto al modello dei successivi elogi d'età augustea ${ }^{93}$, alcuni dei quali presentano un tipo di cornice analoga a quella della nostra iscrizione. Forse potremmo immaginare la nostra lastra applicata ad un basso plinto sorreggente la statua del personaggio, nell'ambito dell'edificio scenico o della vicina porticus.

In ogni caso le implicazioni di carattere storico del nuovo documento epigrafico sono senz'altro notevoli: esso ci assicura che Lepido, al momento dell'uccisione di Cesare, non era magister equitum in perpetuo, ma tertium (o almeno così fu poi ricordato) e dà inoltre conferma della sua terza acclamazione imperatoria, ricevuta forse dopo il suo arrivo in Africa, nell'estate del 40 a.C.

L'iscrizione di Terracina, al pari di quella di Thabraca, dovrebbe in definitiva collocarsi nel ristretto periodo di tempo a cavallo tra l'autunno del 37 e quello del 36 a.C., in occasione del rinnovo del patto triumvirale, quando Lepido sembrava ancora godere di un certo peso politico ed essere utile in vista dell'imminente scontro con Sesto Pompeo. 


\section{BIBLIOGRAFIA}

Allély 2000 = A. Allély, La basilica Aemilia au II et $I^{e r}$ siècles avant Jésus-Christ. Une histoire de famille, in E. Deniaux (a cura di), Rome antique. Pouvoir des images, images du pouvoir. Actes du colloque (Caen 1996), Caen, 2000, p. 137-150.

Allély 2004 = A. Allély, Lépide, le Triumvir, Bordeaux, 2004.

Amela Valverde 2002 = L. Amela Valverde, Lépido en Hispania, in HAnt, 26, 2002, p. 35-58.

Assenmaker $2012=\mathrm{P}$. Assenmaker, Nouvelles perspectives sur le titre d'imperator et l'appellatio imperatoria sous la République, dans Revue belge de philologie et d'histoire, 90-1, 2012, p. 111-142.

Aurigemma - Bianchini - De Santis 1957 = S. Aurigemma, A. Bianchini, A. De Santis, Circeo Terracina-Fondi, Roma, 1957.

Badian 1958 = E. Badian, Foreign clientelae (264-70 B.C.), Oxford, 1958.

Badian 1991 = E. Badian, M. Lepidus and the Second Triumvirate, in Arctos, 15, 1991, p. 5-16.

Bandelli 1987 = G. Bandelli, Per una storia della classe dirigente di Aquileia repubblicana; le iscrizioni da un edificio di spettacolo, in Vita sociale, artistica e commerciale di Aquileia romana, I, Udine, 1987, p. 97-127.

Bastien 2007 = J.-L. Bastien, Le triomphe romain et son utilisation politique à Rome aux trois derniers siècles de la République, Roma, 2007.

Broughton 1952 = T.R.S. Broughton, The Magistrates of the Roman Republic, I-II, New York, 1952. Buonopane - Braito 2016 = A. Buonopane, S. Braito, Le iscrizioni esposte nei teatri romani: aspetti $e$ problemi. Un caso di studio: i sedili di Aquileia, in A. Donati (a cura di), L'iscrizione esposta. Atti del Convegno (Bertinoro 2015), Faenza, 2016, p. 147-188.

Cancellieri $1990=$ M. Cancellieri, $A$ proposito di Cn. Domitius Calvinus e la colonia triumvirale di Tarracina, in La Valle Pontina nell'antichità. Atti del Convegno di studi (Cori 1985), Roma, 1990, p. 45-47. Cassieri $2004=$ N. Cassieri, Il complesso del teatro-portico di Terracina. Prime acquisizioni, in Lazio \& Sabina 2. Atti del Convegno (Roma 2003), Roma, 2004, p. 277-288.

Cassieri 2004-2005 = N. Cassieri, Le indagini nel complesso del teatro-portico di Terracina, in Scienze dell'Antichità, Storia Archeologia Antropologia, 12, 2004-2005, p. 509-525.

Cassieri $2016=$ N. Cassieri, Terracina. Spazi e forme di culto nei contesti urbani, in M. Valenti (a cura di), L'architettura del sacro in età romana. Paesaggi, modelli, forme e comunicazione, Roma, 2016, p. 35-48.

Cassieri - Innico 2007 = N. Cassieri, P.C. Innico, Ritrovamenti lungo la via Appia da Mesa di Pontinia a Terracina, in Lazio \& Sabina 4. Atti del Convegno (Roma 2006), Roma, 2007, p. 207-218.

Cassieri - Innico 2009 = N. Cassieri, P.C. Innico, Terracina. La città bassa. Elementi di topografia urbana, in Lazio \& Sabina 5. Atti del Convegno (Roma 2007), Roma, 2009, p. 369-381.

Cenerini 2016 = F. Cenerini, La donna e il triumviro. Sulla cosiddetta laudatio Turiae, in F. Cenerini, F. Rohr Vio (a cura di), Matronae in domo et in re publica agentes. Spazi e occasioni dell'azione femminile nel mondo romano tra tarda repubblica e primo impero, Trieste, 2016, p. 137-163.

Coarelli 1982 = F. Coarelli, Lazio, Roma, 1982. 
Coarelli 1990 = F. Coarelli, Mutamenti economici e sociali nella Valle Pontina tra media e tarda Repubblica, in La Valle Pontina nell'antichità. Atti del Convegno (Cori 1985), Roma, 1990, p. 51-56.

Cogitore 2002 = I. Cogitore, La légitimité dynastique d'Auguste à Néron à l'épreuve des conspirations, Roma, 2002.

Conticello 1966 = B. Conticello, Scavi a Terracina nel Foro Emiliano, in Archeologia, 4-22, 1966, p. 71-72.

Coppola 1989 = M.R. Coppola, Terracina: il museo e le collezioni. Un catalogo per la realizzazione del Museo Archeologico, Roma, 1989.

Coppola 1993 = M.R. Coppola, Terracina. Il foro emiliano, Roma, 1993.

de La Blanchère 1884 = M.-R. de La Blanchère, Terracine. Essai d'histoire locale, Paris, 1884.

Eck 1996 = W. Eck, Tra epigrafia, prosopografia e archeologia. Scritti scelti, rielaborati ed aggiornati, Roma, 1996.

Ferriès 2007 = M.-Cl. Ferriès, Les partisans d'Antoine, Bordeaux, 2007.

Fishwick 1994 = D. Fishwick, On the origins of Africa Proconsularis II, in AntAfr, 30, 1994, p. 57-80.

Gorostidi Pi - Núñez Marcén 2016 = D. Gorostidi Pi, J. Núñez Marcén, Un frammento dei Fasti consulares dal Foro di Tusculum, in ZPE, 199, 2016, p. 223-230.

Gowing 1992 = A.M. Gowing, The triumviral narrative of Appian and Cassius Dio, Ann Arbor, 1992.

Gregori - Almagno 2019 = G.L. Gregori, G. Almagno, Roman calendars: Imperial birthdays, victories and triumphs, Beau Bassin, 2019.

Guey 1957 = J. Guey, Dédicace à Lépide (37-36 av.J.-C.) trouvée à Tabarka (Tunisie), in BSNAF, 1957, p. 186-188.

Guey - Pernette 1958 = J. Guey, A. Pernette, Lépide à Thabraca, in Karthago, 9, 1958, p. 80-89.

Hayne 1974 = L. Hayne, The defeat of Lepidus in 36 B.C., in Acta Classica, 17, 1974, p. 59-65.

Jehne $2015=$ M. Jehne, From Patronus to Pater, the changing role of patronage in the period of transition from Pompey to Augustus, in F. Pina-Polo, M. Jehne (a cura di), Foreign clientelae in the Roman Empire. A reconsideration, Stuttgart, 2015.

Jordan 2015 = B. Jordan, The Fasti Consulares Capitolini and Caesar's magistri equitum designati, in ZPE, 196, 2015, p. 231-239.

Letta 2017 = C. Letta, Fasti Albenses: progressi e palinodie sui Fasti Consulares, in S. Segenni, M. Bellomo (a cura di), Epigrafia e politica. Il contributo della documentazione epigrafica allo studio delle dinamiche politiche nel mondo romano, Milano, 2017, p. 27-64.

Lipps 2016 = J. Lipps, Statuen kniefaelliger Orientalen aus Rom und ein Dreifuss im Olympieion von Athen, in $\operatorname{MDAI}(R), 122,2016$, p. 203-252.

Lugli 1926 = G. Lugli, Anxur-Tarracina, Roma, 1926 (Forma Italiae, I, 1).

Melchor Gil 2017 = E. Melchor Gil, Los origenes del patronato civico en las provincias hispanas: desde Cneo Pompeyo Magno al triunviro Marco Emilio Lépido, in RSA, 47, 2017, p. 35-58.

Melchor Gil 2018 = E. Melchor Gil, El patronato cívico en la Hispania romana, Sevilla, 2018.

Mesolella 2012 = G. Mesolella, La decorazione architettonica di Minturnae Formiae Tarracina: l'età augustea e giulio-claudia, Roma, 2012. 
Paci 2014 = G. Paci, Liberte e schiave a teatro e all'anfiteatro nel Piceno, in C. Zaccaria (a cura di), L'epigrafia dei porti, Trieste, 2014, p. 275-288.

Pina Polo 2018 = F. Pina Polo, Magistrates without pedigree: the consules suffecti of the Triumviral Age, in JRS, 108, 2018, p. 99-114.

Roddaz 2003 = J.-M. Roddaz, Octavien-Auguste et les soldats perdus de Lépide, in J.-P. Bost, J.M. Roddaz, F. Tassaux (a cura di), Mélanges offerts à Louis Maurin. Itinéraire de Saintes à Dougga, Bordeaux, 2003, p. 189-203.

Rohr Vio 2004 = F. Rohr Vio, Marco Emilio Lepido tra memoria e oblio nelle Historiae di Velleio Patercolo, in RCCM, 46-2, 2004, p. 277-301.

Rohr Vio 2007 = F. Rohr Vio, Recensione a Allely 2004, in RCCM, 49, 2007, p. 173-176.

Ronchi 2017 = D. Ronchi, La colonia di Circeii. Dal tardo arcaismo alla colonia di Cesare padre: santuari ed evidenze monumentali, Pisa, 2017.

Schneider 1986 = R.M. Schneider, Bunte Barbaren. Orientalenstatuen aus farbigen Marmor in der römischen Repräsentationskunst, Worms, 1986.

Schumacher 1985 = L. Schumacher, Die imperatorischen Akklamationen der Triumvirn und die auspicia des Augustus, in Historia, 34-2, 1985, p. 191-222.

Simpson 2006 = C.J. Simpson, M. Aemilius Lepidus, in Latomus, 65-3, 2006, p. 628-633.

Solin 1981 = H. Solin, Caesar und P. Clodius Pulcher in Terracina, in ZPE, 43, 1981, p. 357-361.

Syme 1993 = R. Syme, L'aristocrazia augustea (trad. it.), Milano, 1993.

Tansey 2008 = P. Tansey, Q. Aemilius Lepidus (Barbula?) cos. 21 B.C., in Historia, 57-2, 2008, p. 174-207.

Tansey $2018=$ P. Tansey, Notabilia varia in the Fasti of Alba Fucens, in SCO, 64, 2018, p. 199-269.

Weigel $1974=$ R. Weigel, Lepidus reconsidered, in Acta Classica, 17, 1974, p. 67-73.

Weigel $1985=$ R. Weigel, Augustus' Relations with the Aemilii Lepidi - Persecution and Patronage, in RhMus, 128, 1985, p. 180-191.

Weigel $1992=$ R. Weigel, Lepidus. The tarnished triumvir, London, 1992.

Welch 1995 = K. Welch, The career of M. Aemilius Lepidus 49-44 B.C., in Hermes, 123, 1995, p. 443-454.

Welch 2012 = K. Welch, Magnus Pius. Sextus Pompeius and the Transformation of the Roman Republic, Swansea, 2012.

Zevi 2016 = F. Zevi, $I$ Fasti di Privernum, in ZPE, 197, 2016, p. 287-309.

Zevi 2017 = F. Zevi, I Fasti di Privernum alla luce della collaborazione con Filippo Càssola, in M. Chiabà, L. Cristante (a cura di), Il sussurro di una brezza leggera. Ricordo di Filippo Càssola, Trieste, 2017, p. 5-18.

Zucca 1996 = R. Zucca, Inscriptiones latine liberae rei publicae Africae, Sardiniae et Corsicae, in L'Africa romana, XI, Ozieri, 1996, p. 1425-1489.

\section{NOTE}

1. In questo paragrafo si riprende in maniera sintetica quanto già scritto in precedenza (Cassieri 2004; Cassieri 2007), con integrazioni e aggiornamenti al 2017, forniti dalle acquisizioni derivanti dalle attività di sistemazione e restauro dei resti murari e dei 
materiali riportati in luce. Da allora è stata completata la demolizione già in fase avanzata dei caseggiati moderni impiantati l'uno sulla cavea, nel settore centroorientale, l'altro nell'area della porticus post scaenam. È in preparazione l'edizione scientifica a più mani dei risultati conseguiti in oltre vent'anni di ricerche dirette da chi scrive (1994-2017) per conto della ex Soprintendenza archeologica del Lazio, sia per quanto riguarda le strutture monumentali, che gli elementi architettonici e di arredo e le iscrizioni. In questo contributo si presentano alcuni dei reperti più rilevanti rinvenuti nel complesso.

2. Lugli 1926; molte informazioni erano state rese note già da de La Blanchère 1884, che aveva soggiornato nel territorio pontino negli anni 1879-1881; più recentemente Cassieri - Innico 2007; Cassieri - Innico 2009.

3. Coarelli 1982, p. 316.

4. Ipotesi avanzata da Coarelli 1982, p. 165 sq.; Coarelli 1990, p. 54-55, sulla base del bollo Cn. Domitius Calvinus coloneis dedit (CIL X, 6314), databile nella seconda metà del I sec. a.C., per il quale accoglie una provenienza da Terracina, rivelatasi errata (Cancellieri 1990, p. 45-46). Sui bolli di Domizio Calvino e il loro rapporto con la colonia di Circei: Ronchi 2017, p. 88-90 e n. 336.

5. Mesolella 2012 p. 64 e 354-355.

6. Tra di essi va ricompreso anche il Tempio a tre celle/cd. Capitolium, realizzato all'epoca del secondo triumvirato, che, sebbene posto non all'interno dello spazio forense ma nelle immediate vicinanze, è ad esso funzionalmente collegato. La datazione intorno al 42 a.C. proposta da Lugli 1926, col. 84 viene comunemente accettata.

7. CIL X, 6306 = EDR156631. Sulle fasi di ritrovamento di questo eccezionale lastricato tra il 1846 e il 1959, vd. Coppola 1993, p. 26-40.

8. I modi e le forme del coinvolgimento degli illustri committenti non sono noti; spesso agivano «attraverso clienti e personaggi di fiducia di estrazione locale», come il citato Aulus Aemilius, sovvenzionatore anche del Tempio a Roma e Augusto (CIL X, $6305=$ EDR156629), il cui rapporto con la potente famiglia senatoria degli Aemilii Lepidi è suggestivo ma tutto da dimostrare (Mesolella 2012, p. 84-90 e 658-667).

9. Per la pronta ricezione degli orientamenti in voga, valgano a titolo esemplificativo le strette affinità tecniche e architettoniche, oltre che decorative, del Tempio Maggiore di Terracina con quello coevo «di Augusto» a Puteoli e per il pavimento marmoreo della cella, con l'Aula del Colosso del Foro di Augusto a Roma: Cassieri 2016, p. 35-42.

10. Aurigemma - Bianchini - De Santis 1957, p. 28. Solo negli anni Sessanta fu possibile all'allora Soprintendenza di Roma I condurre sondaggi molto circoscritti in un'area libera che evidenziarono avanzi dell'aditus orientale (Conticello 1966, p. 71-72).

11. Vd. riferimenti bibliografici supra.

12. In opera incerta sono il muraglione presente nell'ala est, i due aditus maximi, il muro di contenimento della cavea orientale, quello di spalla dell'ambulacro superiore e il muro della scaenae frons che si addossa al muro di fondo della porticus realizzato in precedenza nella medesima tecnica edilizia. La presenza di alcuni graffiti nel lato posteriore del muraglione che chiude il braccio orientale del portico post scaenam, tra cui uno menzionante la morte di Clodio (CIL I $\left.{ }^{2}, 3105\right)$, avvenuta nel 52 a.C., fornisce un importante punto di riferimento cronologico (cf. Solin 1981). 
13. Un settore centrale della porticus, gli aditus maximi, porzioni del versante orientale della cavea e dell'orchestra, oltre a circa la metà dell'edificio scenico; dei numerosi reperti, attualmente in corso di studio, solo una parte è stata finora sottoposta a catalogazione e restauro.

14. Per lo schema disegnativo del pavimento basato sull'incrocio di fasce di marmo bianco che delimitano tappeti di grigio bardiglio e per la tipologia delle colonne con fusti rudentati con «Segmentstab-Kanneluren»: Cassieri 2004, p. 287 n. 19 e 21. Di diverso avviso Mesolella 2012, p. 342-344, che, considerata la semplificazione del rilievo, propone di anticipare la datazione delle colonne già in tarda età augustea o giulio-claudia.

15. Il diametro massimo ricostruito del teatro è di $61,70 \mathrm{~m}$ circa. La cavea era divisa in sei cunei da sette scalette radiali.

16. Il blocco, rinvenuto tra la prima scaletta radiale e il tribunal nel 2007 e rimasto finora inedito, è accumulato insieme ad altri pesanti materiali lapidei in un settore dell'area di cantiere al momento inaccessibile per ragioni di sicurezza; sui loca in generale da ultimi: Paci 2014; Buonopane - Braito 2016.

17. Una ricca liberta di nome Fufia Viticula è attestata dal grande titulus relativo a un edificio funerario eretto nella prima metà del I sec. d.C. in località Valle: $A E$ 2007, 352.

18. Nel 2017 uno di questi fusti, trovato integro nell'area dell'orchestra anche se con notevoli lesioni, è stato ricollocato sulla prima base a est dopo un impegnativo intervento di consolidamento, restauro.

19. Devo queste osservazioni a D. Nepi, che nella sua tesi di specializzazione (2011-2012), relatore C.F. Giuliani, in corso di stampa, ha approfondito l'aspetto della ricostruzione tridimensionale del teatro di Terracina con particolare riguardo all'edificio scenico e al funzionamento del sipario.

20. Aurigemma - Bianchini - De Santis 1957, p.15; si veda per esempio l'impianto termale presso l'area AGIP, sul margine sinistro di via Roma, nel centro urbano: Cassieri - Innico 2009, p. 375-379.

21. La scultura, complessivamente alta $2,40 \mathrm{~m}$, dopo un impegnativo intervento di restauro conclusosi nel 2017, è stata presentata da chi scrive al XII Convegno Internazionale ASMOSIA (Izmir 2018), i cui Atti sono in preparazione.

22. Schneider 1986.

23. Schneider 1986, p. 201-202; Lipps 2016, p. 231-237.

24. Cassieri 2016, p. 42-44 segnala come confronto immediato, per contesto di ritrovamento, tipologia di monumento e decorazione simbolica allusiva ad Apollo, uno dei quattro altari in marmo bianco rinvenuti nel teatro di Arles, costruito nel 25-10 a.C. (l'esemplare detto «dei cigni»), privo tuttavia di dedica e originariamente collocato nell'orchestra.

25. Cassieri 2016, p. 43-44.

26. InscrIt., XIII, 1, 31, 36; CIL X, 6087 = EDR152842: triump(hauit) ex Raetis... Invece negli elogi augustei, a Roma e nelle varie città d'Italia, ricorre la forma con l'aspirata.

27. Nei fasti trionfali (InscrIt., XIII, 1, 36) si fa riferimento al secondo trionfo di Lepido (per il quale vd. infra) con l'espressione: M(arcus) Aemilius Lepidus iterum ex Hispania / prid(ie) K(alendas) Ian(uarias) [tr]iumphauit; palmam de[dit]. 
28. CIL VI, 41062 = EDR093344: ... [quom abs te ---] de restitutione mea M. L[epi]dus conlega praesens interp[ellaretur et ad eius] pedes prostrata humi [n]on modo non adleuata, sed tra[cta et seruilem in] modum rapsata, liuori[bus c]orporis repleta: Cenerini 2016. Vista la rarità delle dediche per Lepido Allély 2004, p. 241-243 ipotizza vi sia stata nei suoi confronti una sorta di damnatio memoriae; ben diversa fu tuttavia la sorte di M. Antonio, il cui nome fu scalpellato dalle iscrizioni.

29. IRC III, 31, secondo le integrazioni proposte da Melchor Gil 2017, p. 53-54; Melchor Gil 2018, p. 44-46: [M(arco) Aem(ilio) Lepido ] / [pontif(ici)] $m$ [ax(imo, co(n)s(uli)], [imp(eratori) $p]$ atr[ono].

30. AE 1959, 77 = ILLRP $1276(31,5 \times 27 \times 4,8 \mathrm{~cm}$; lett. 4,3-2,2 cm); cf. Weigel 1992, p. 82-83; Zucca 1996, p. 1447-1448 n. 32; Allely 2004, p. 180-181.

31. Elenco completo delle cariche in RE 73; PIR A 367; Broughton 1952, p. 527.

32. Di avviso contrario era Badian 1991, ma vedi le obiezioni di Allély 2004, p. 180.

33. Fonti in Broughton 1952, p. 333. Lepido faceva parte del collegio dei pontefici da prima del 57 a.C. (Broughton 1952, p. 205-206).

34. App., BC, 2, 21; Plut., Ant., 6, 4; Broughton 1952, p. 257; Weigel 1974, p. 68; Welch 1995; Rohr Vio 2007, p. 175-176.

35. Badian 1991. Se nulla si è conservato per Antonio, a causa anche della sua damnatio memoriae, per Ottaviano si vedano ILLRP 416 (Saticula): C(aio) Iulio C(ai) f(ilio) Caesari / imp(eratori) triumviro r(ei) p(ublicae) c(onstituendae) / patrono / d(ecreto) d(ecurionum); $A E$ 1989, 342b (Siracusa): [C(aio) Iulio Caesari] imp (eratori) div [i f(ilio)] / [triumuiro r(ei) $p$ (ublicae) $c$ (onstituendae) st]atua dic(ata). L'iterazione del triumvirato per Ottaviano è attestata solo nella iscrizione commemorante la costruzione delle mura di Tergeste (InscrIt., X, 4, 20-22; ILLRP 418, a. 33 a.C.) e sulle monete ( $R R C$ n. 538, 540). Antonio a quanto pare non indicò l'iterazione. Per le tre acclamazioni imperatorie di Antonio e Ottaviano: RRC n. 533-534.

36. InscrIt., XIII, 2, 44, su cui ora Gregori - Almagno 2019, p. 69-70. Cf. Hayne 1974, con richiamo ai passi di Appiano e Cassio Dione, ma soprattutto Gowing 1992, p. 123-142, che discute la diversa ricostruzione dei fatti nei due autori; Rohr Vio 2004, a sua volta, sottopone a critica il ritratto di Lepido in Velleio, che mira a squalificare l'immagine del triumviro nelle sue capacità militari e a inficiare la sua credibilità quale possibile erede di Cesare; sulla parzialità delle nostre fonti nei riguardi di Lepido insiste anche Weigel 1974.

37. Weigel 1992, p. 94-100; Allély 2004, p. 187-192; Welch 2012, p. 278 n. 59. App., BC, 5, 124-126, 512-524 e Dio., 49, 12, 2-3. Così Suet., Aug., 16 sunteggia la caduta di Lepido: Post Pompei fugam collegarum alterum $M$. Lepidum, quem ex Africa in auxilium euocarat, superbientem uiginti legionum fiducia summasque sibi partes terrore et minis uindicantem spoliauit exercitu supplicemque concessa uita Circeios in perpetuum relegauit. Numerose sono le testimonianze archeologiche attribuibili a ville d'età romana nel comprensorio del Circeo, la cui costruzione per confronto con analoghe strutture del litorale laziale si può collocare tra la seconda metà del II sec. a.C. e la seconda metà del I sec. a.C., ma nessuna è riferibile agli Aemilii Lepidi: Ronchi 2017, p. 129-131.

38. App., BC, 5, 95, 398; Dio., 48, 54, 6.

39. Weigel 1992, p. 84-85 immagina un importante ruolo di Lepido nella creazione della provincia d'Africa con capitale Cartagine e nella distribuzione di terre a coloni provenienti dall'Italia, dove erano stati privati delle loro proprietà; anche Allély 2004, 
p. 177-192 sottolinea come tra il 40 e il 36 a.C. le fonti letterarie non menzionino praticamente più Lepido; per l'amministrazione dell'Africa da parte di Lepido vd. infra.

40. Liv., 40, 51, 2, a proposito dell'operato del censore del 179 a.C. M. Aemilius Lepidus, accusato di aver costruito con fondi pubblici ma per fini privati una diga o un molo (molem), ad Tarracinam, dove egli aveva praedia; secondo Coarelli 1990, p. 53 essi sarebbero da localizzare a S del Pisco Montano; la struttura avrebbe permesso il passaggio intorno alla rupe prima del famoso taglio, per favorire il trasporto dei prodotti dai suoi fondi al porto. Egli non fu l'unico senatore presente a Terracina nel corso del II sec. a.C.: anche i Sulpicii Galbae dovevano avere interessi sul posto, se il console del 144 a.C. Ser. Sulpicius Ser.f. Galba appaltò la messa in opera di un pavimento musivo, collaudando poi il lavoro (ILLRP 338). Nella prima metà del I sec. a.C. ricevettero onori a Terracina i legati senatorii Manius Sabidius Mani f. (dai cittadini Romani che svolgevano attività commerciali a Panhormus) e M. Favonius M.f. (dagli abitanti di Agrigentum): ILLRP 387, 398. Nella seconda metà del I sec. a.C. un esponente dei Livii Ocellae fu onorato a Terracina forse dagli Hispalenses: CIL X, 6319, su cui Coppola 1989, p. 52 nr. 28; Eck 1996, p. 147-154.

41. Zevi 2016, p. 295-303; Zevi 2017.

42. Zevi 2017, p. 15-16; cf. Jordan 2015.

43. È il caso anche del recentissimo frammento dei Fasti Consolari rinvenuti nel foro di Tusculum: Gorostidi Pi - Núñez Marcén 2016.

44. InscrIt., XIII, 1, 1b: C(aius) Iulius C(aii) f(ilius) C(aii) n(epos) Caes[a]r IIII abd(icauit) [dict(ator) rei gerundae causa], M(arcus) Aemilius M(arci) [f(ilius) Q(uinti) n(epos) Lepidus II abd(icauit) mag(ister) eq(uitum); $C$ (aius) Iulius $C$ (aii) f(ilius) $C$ (aii) $n$ (epos) Caesar in perpetuum dic(tator) rei gerundae causa, M(arcus) Aimilius M(arci) f(ilius) Q(uinti) n(epos) Lepidus III mag(ister) eq(uitum)].

45. In effetti un errore di incisione in un documento di carattere ufficiale come i Fasti potrebbe sembrare sorprendente, ma in realtà anche in testi di carattere giuridico errori di esecuzione e duplicazioni non sono fenomeni rari.

46. Dopo l'edizione di Letta 2017, si vedano ora le puntualizzazioni di Tansey 2018.

47. Bastien 2007, p. 246-247 ; Assenmaker 2012, p. 130-134.

48. Amela Valverde 2002.

49. RE 70 ; Tr. Pl. 49 (Broughton 1952, p. 259).

50. Ps. Caes., Bell. Alex., 48-55 ; Val. Max., 9, 4, 2 ; Dio., 42, 15. Allély 2004, p. 49-50.

51. Dio., 43, 1, 2-3.

52. Badian 1958, p. 278-282 ; Jehne 2015, p. 303-306.

53. Pour les six premiers mois de l'année, Lépide gouverne en effet sa province par l'entremise de légats. Vell., 2, 63, 1 ; App., BC, 2, 107 ; Dio., 43, 51, 8.

54. Charge que Lépide obtient après les ides de mars, grâce au soutien d'Antoine. App., BC, 2, 132 ; Dio., 44, 53, 6 ; Vell., 2, 63, 1 ; Liv., Per., 117 ; Allély 2004, p. 215-218.

55. Sur la consuetudo de l'appellatio par la Sénat, voir Assenmaker 2012, p. 133-138.

56. Cic., Phil., 5, 40 ; 41. CIL I², p. 50 (Fastes triomphaux du Capitole). Ces récompenses sont demandées par Cicéron, ce dont Lépide a conscience (Cic., Fam., 10, 34a, 1). Syme 1993, p. 165 et Allély 2004, p. 92 et 94-96. 
57. Weigel 1992, p. 68 ; App., BC, 4, 2, 7 ; Dio., 46, 55, 4 et Allély 2004, p. 122-123. Les triumvirs gouvernent leurs provinces par l'entremise de légats.

58. Selon Weigel 1992, p. 79, Lépide n'aurait été utile qu'en cas de défaite des triumvirs. Allély 2004, p. 143-145.

59. App., BC, 4, 3, 9 .

60. Dio., $48,1,2-3$.

61. App., $B C, 5,3,12$.

62. App., $B C, 5,12,47$.

63. Weigel 1992, p. 80.

64. Dio., 48, 20, 4.

65. App., BC, 5, 53, 223 ; Allély 2004, p. 147-148.

66. App., BC 5, 65, 274 ; Dio., 48, 28, 4 ; Weigel 1992, p. 82 et Allély 2004, p. 177. Dans leur récit des négociations entre Antoine et Octavien, les sources évoquent succinctement le sort de Lépide. Toutefois, aussi bien Appien que Dion ne font part que d'un "gouvernement » de Lépide et non une possession. Cette confirmation prend les atours d'une simple nomination d'un magistrat, comme c'est fréquemment le cas à la période triumvirale. Si, sur un plan strictement formel, Lépide est l'égal de ses collègues, il reste marginalisé. Ainsi, en 39 à Misène, sa position est si fragile que Sextus Pompée demande à prendre sa place au sein du triumvirat (App., BC, 5, 71, 299). Si Antoine et Octavien refusent, c'est certainement plus par volonté de ne pas faire de Sextus un des leurs plutôt que de soutenir Lépide.

67. En effet, il y a sans doute un délai entre l'arrivée de Lépide dans la province et l'officialisation conjointe, par Antoine et Octavien, de son autorité. Durant ce délai, l' Africa appartient à Antoine.

68. Sur Sextius : RE 13 ; CIL X, 6104 ; Pr. 45 (Broughton 1952, p. 307). Voir aussi Ferriès 2007, p. 468-470. Pour les événements en Afrique avant l'arrivée de Lépide : Dio., 48, 21-23 et Allély 2004, n. 88 p. 144.

69. Dio., $48,23,5$.

70. RE 3 ; cos. design. 29 (Broughton 1952, p. 384).

71. App., BC, 5, 75, 321 et Weigel 1992, p. 82.

72. Sur l'administration de l'Afrique par Lépide, cf. Fishwick 1994, p. 58-64.

73. Dio., $48,46,2$.

74. App., BC, 5, 95, 396-399 et Dio., 48, 54, 4.

75. Appien, dans une épitaphe attenante à la mort politique de Lépide, évoque le grand

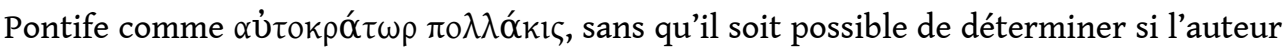
alexandrin fait référence en l'espèce à une troisième acclamation de Lépide (App., $B C, 5$, $126,524)$.

76. Mentionnée plus haut : ILLRP 1276.

77. Badian 1991, p. 5-16, évoque la possibilité que Lépide ait pu, par cette inscription, obliger Antoine et Octavien à l'accepter comme Triumvir, en dépit de son absence à Tarente. Une autre possibilité est que Lépide soit effectivement confirmé dans ses fonctions en 37 sans que cela soit rapporté par les sources. Toutefois il ne nous appartient pas de trancher entre ces deux hypothèses ici. Pour ceci, voir Allély 2004, p. 180.

Mélanges de l'École française de Rome - Antiquité, 131-2 | 2019 
78. Pour une étude sur ce point, voir Guey - Pernette 1958, p. 87 et Weigel 1992, p. 82.

79. L'adverbe le plus courant en l'occurrence aurait été iterum. Nous sommes en présence d'une des deux variantes stylistiques de l'inscription, l'autre portant sur le nom de Lépide, qui ne comporte ni le gentilice, ni la filiation du grand Pontife. Guey Pernette 1958, p. 83.

80. Broughton 1952, p. 359.

81. Guey - Pernette 1958, p. 83 et Guey 1957, p. 186-7.

82. Roddaz 2003, p. 192 n. 45.

83. « Some scuffle », Weigel 1992, p. 82.

84. Idem.

85. Même si une argumentation ex silentio est toujours fragile, il nous semble que ce caslà soit probable.

86. Dio., $48,23,5$.

87. App., $B C, 5,75,321$.

88. Cette hypothèse a été formulée par Schumacher 1985, p. 202. Il y évoque notamment le fait que l'affrontement entre Lépide et Sextius pourrait n'avoir débouché sur aucun combat, ce qui pourrait être probable.

89. Roddaz 2003, p. 192 n. 45.

90. Il primo, M. Aemilius Lepidus, fu giustiziato nel 30 a.C. per ordine di Ottaviano, perché aveva congiurato contro di lui ( $R E 74$ ); Cogitore 2002, p.55-62; dell'altro, il supposto Q. Aemilius Lepidus, padre del console dell'11 d.C. poi proconsole d'Asia sotto Tiberio $(R E 79,63)$, non conosciamo la carriera e la sua stessa esistenza è ora messa in dubbio da J. Morgan: Weigel 1985; Allély 2004, p. 243-244; cf. Tansey 2008.

91. Si deve comunque notare che la colonia Victrix Iulia Lepida nella Hispania Citerior venne ribattezzata colonia Victrix Iulia Celsa: Amela Valverde 2002.

92. Weigel 1985, p. 188-189; Rohr Vio 2004, p. 252; Zevi 2017, p. 14-16.

93. Se ne veda la nuova edizione di G. Alföldy, in CIL VI, 40912-41025.

\section{RIASSUNTI}

Gli scavi condotti nel teatro di Terracina negli anni 2004-2017 hanno restituito molti materiali di rilevante interesse storico e archeologico, in particolare una statua di barbaro «Orientale» in giallo antico, un'ara per Iuppiter Axur e numerosi frammenti che compongono quasi per intero un'iscrizione con la carriera del triumviro Lepido, unico caso finora noto in Italia. Dopo la presentazione del contesto di scavo, si dà qui la prima edizione dell'eccezionale documento, inquadrabile tra il 37 e il 36 a.C., dal quale apprendiamo, in particolare, che nel 44 a.C. Lepido aveva rivestito per la terza volta la carica di magister equitum di Cesare.

The excavations in Terracina's ancient theater from 2004 to 2017 have revealed numerous materials of great historical and archaeological interest. Are highlighted a marmor numidicum 
statue representing an «Oriental» barbarian, an altar dedicated to Iuppiter Axur and numerous fragments which enable to reconstruct almost completely an inscription with the career of the triumvir M. Aemilius Lepidus, a unique case in Italy. After the presentation of the context of the excavation, the paper gives the first analysis of this exceptional document, datable between the years 37 and $36 \mathrm{BC}$, from which we learn that Lepidus was Cesar's magister equitum for the third time in $44 \mathrm{BC}$.

\section{INDICE}

Keywords : M. Aemilius Lepidus triumvir, Emilian Forum, latin inscriptions, roman theatre, Terracina

Parole chiave : M. Aemilius Lepidus triumviro, Foro Emiliano, iscrizioni latine, Terracina, teatro romano

\section{AUTORI}

\section{NICOLETTA CASSIERI}

Soprintendenza Archeologia, Belle Arti e Paesaggio per le province di Frosinone, Latina e Rieti nc.icaro@gmail.com

\section{GIAN LUCA GREGORI}

Sapienza Università di Roma - gianluca.gregori@uniroma1.it

\section{JEAN-BAPTISTE REFALO-BISTAGNE}

Aix-Marseille Université, Sapienza Università di Roma - jean.baptiste.rb@live.fr 